UCRL-ID- 129782

\title{
Advanced Accelerator Theory Development
}

\author{
S. E. Sampayan, T. L. Houck, B. Poole, N. Tishchenko, P. A. Vitello, and L. Wang
}

Final Report

February 9, 1998

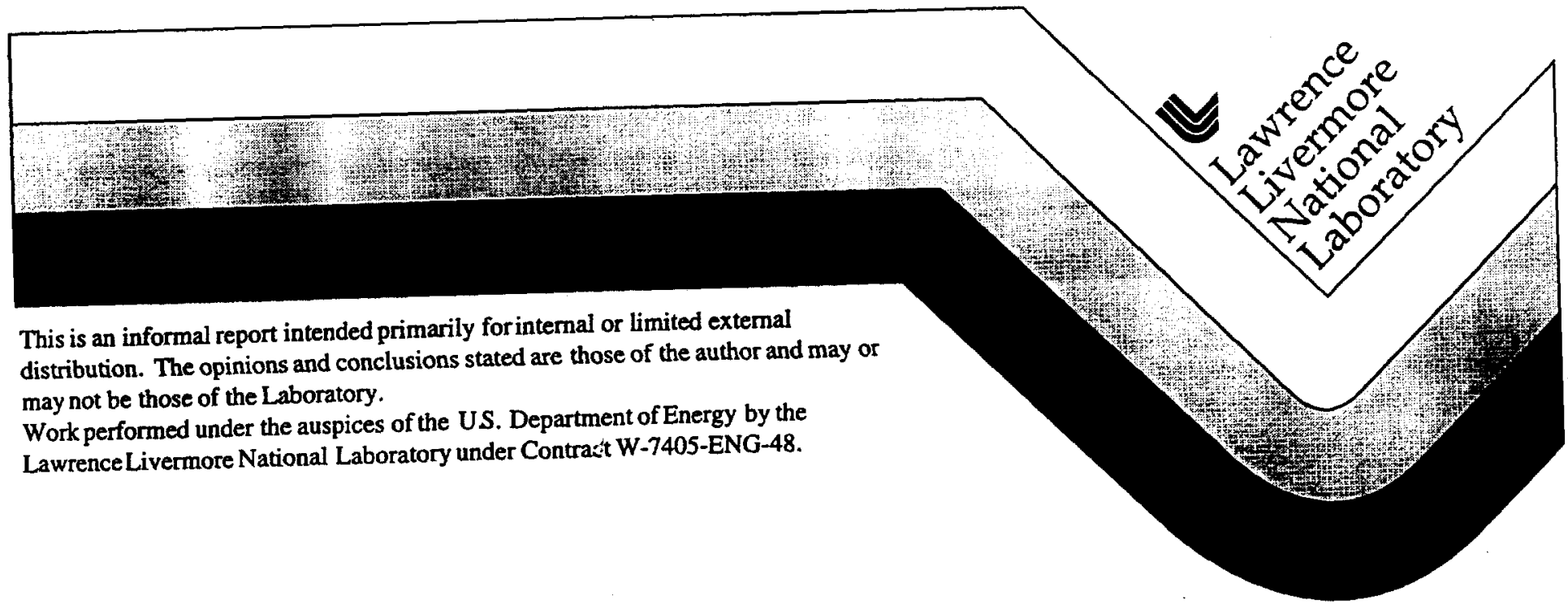




\section{DISCLAIMER}

This document was prepared as an account of work sponsored by an agency of the United States Government. Neither the United States Government nor the University of California nor any of their employees, makes any warranty, express or implied, or assumes any legal liability or responsibility for the accuracy, completeness, or usefulness of any information, apparatus, product, or process disclosed, or represents that its use would not infringe privately owned rights. Reference herein to any specific commercial product, process, or service by trade name, trademark, manufacturer, or otherwise, does not necessarily constitute or imply its endorsement, recommendation, or favoring by the United States Government or the University of California. The views and opinions of authors expressed herein do not necessarily state or reflect those of the United States Government or the University of California, and shall not be used for advertising or product endorsement purposes.

This report has been reproduced directly from the best available copy.

Available to DOE and DOE contractors from the Office of Scientific and Technical Information

P.O. Box 62, Oak Ridge, TN 37831

Prices available from (615) 576-8401, FTS 626-8401

Available to the public from the

National Technical Information Service

U.S. Department of Commerce

5285 Port Royal Rd.,

Springfield, VA 22161 


\title{
Advanced Accelerator Theory Development ${ }^{*}$
}

\author{
S. E. Sampayan, T.L. Houck, B. Poole, N. Tishchenko, P. A. Vitello, and L. Wang \\ Lawrence Livermore National Laboratory \\ P. O. Box 808 \\ Livermore, CA 94551
}

\begin{abstract}
$\underline{\text { Abstract }}$
A new accelerator technology, the dielectric wall accelerator (DWA), is potentially an ultra compact accelerator/pulsed power driver. This new accelerator relies on three new components: the ultra-high gradient insulator, the asymmetric Blumlein, and low jitter switches. In this report, we focused our attention on the first two components of the DWA system: the insulators and the asymmetric Blumlein. First, we sought to develop the necessary design tools to model and scale the behavior of the high gradient insulator. To perform this task, we concentrated on modeling the discharge processes (i.e., initiation and creation of the surface discharge). In addition, because these high gradient structures exhibit favorable microwave properties in certain accelerator configurations, we performed experiments and calculations to determine the relevant electromagnetic properties. Second, we performed circuit modeling to understand energy coupling to dynamic loads by the asymmetric Blumlein. Further, we have experimentally observed a non-linear coupling effect in certain asymmetric Blumlein configurations. That is, as these structures are stacked into a complete module, the output voltage does not sum linearly and a lower than expected output voltage results. Although we solved this effect experimentally, we performed calculations to understand this effect more fully to allow better optimization of this DWA pulse-forming line system.
\end{abstract}

*Work performed under the auspices of the US Dept. of Energy by LLNL under contract W-7405-ENG-48. 


\title{
Advanced Accelerator Theory Development
}

\author{
S. E. Sampayan, T. L. Houck, B. Poole, N. Tishchenko, P. A. Vitello, and L. Wang \\ Lawrence Livermore National Laboratory \\ P. O. Box 808 \\ Livermore, CA 94551
}

\section{INTRODUCTION}

A new accelerator technology, the dielectric wall accelerator (DWA), is potentially an ultra compact accelerator/pulsed power driver [1]. Because of its compact size (which translates into lower cost), it has potential applications in several defense missions and in the commercial sector. It utilizes three principal components to achieve a high gradient in a compact structure. These components include the insulator, the asymmetric Blumlein, and the switches.

A primary component that limits the acceleration gradient in the DWA is the vacuum insulator. We have expanded on the initial work [2], and further studied and developed this component over the past several years $[3,4]$. For short pulse systems, these structures have withstood $20 \mathrm{MV} / \mathrm{m}$ gradients in the presence of a plasma cathode and a $1 \mathrm{kA}$ electron beam.

The asymmetric Blumlein is a novel method for generating a fast electrical pulse in a single step process (Figure 1). The system consists of a stacked series of these circular modules and generates a high voltage when switched. Each Blumlein is composed of two dielectric layers with differing permittivities. On each surface and between the dielectric layers are conductors that form two parallel plate radial transmission lines. The lower permittivity side of the structure is referred to as the slow line. The higher permittivity side of the structure is referred to as the fast line.

Operation of the Blumlein is as follows. The center electrode between the fast and slow line is initially charged to a high potential. Because the two lines have opposite polarities, there is no net voltage across the inner diameter (ID) of the Blumlein. Upon applying a closure across the outside of the structure by a surface flashover or similar switch, two reverse polarity waves are initiated which propagate radially inward towards the ID of the Blumlein. The wave in the fast line reaches the ID of the structure prior to the arrival of the wave in the slow line. When the fast wave arrives at the ID of the structure, the polarity there is reversed in that line only, resulting in a net voltage across the ID of the asymmetric Blumlein. This high voltage will persist until the wave in the slow line finally reaches the ID. In the case of an accelerator, a charged particle beam can be injected and accelerated during this time. In the case of a compact pulsed power driver, a stalk can be placed on axis that will sum the voltage developed by each module. 


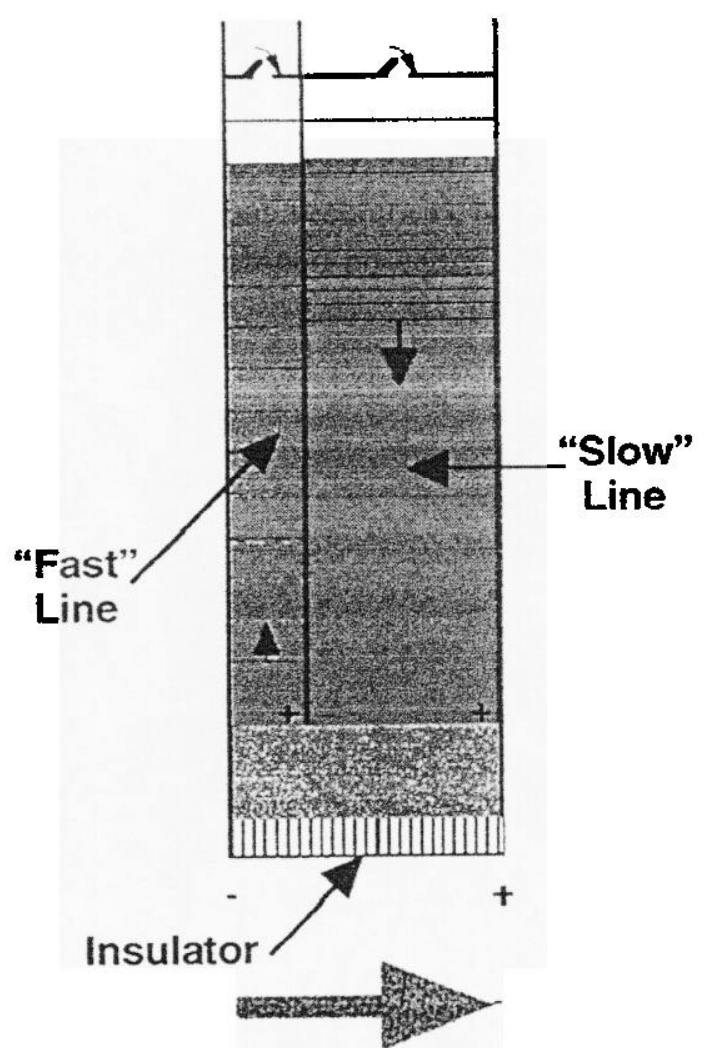

Figure 1 - Dielectric Wall Accelerator Asymmetric Blumlein Module.

Typical materials used for the dielectric wall accelerator are common microwave laminates (RTDuriod, Rogers Corporation, $\varepsilon_{\mathrm{r}}=10$ ) and $\mathrm{TiO}_{2}$. Commercially available $\mathrm{TiO}_{2}$ suffers from the disadvantage of high loss, an abnormally low dielectric constant, and in particular, poor high voltage breakdown characteristics. Use of purer material and the addition of proper dopants, however, allowed achievement of close to theoretical permittivity values $\left(\varepsilon_{\mathrm{r}} \sim 100\right)$ and breakdown fields exceeding $200 \mathrm{kV} / \mathrm{cm}$ (Figure 2) [5]. Higher permittivity materials $\left(\varepsilon_{\tau}>1000\right)$ also show promise and the effect of slight material non-linearities are under investigation.

Single switches, which we have developed, have shown sub-nanosecond jitter at the required gradients (Figure 3) [6]. These switches consist of an insulator system on the periphery of the DWA structure upon which a surface flashover is initiated by a prompt ultraviolet pulse. Sixty such sites are used in the present DWA module. In the present geometry, splitting of the single output beam from the present laser system is done with a specialized multi-lens and fiberoptics array. For simplicity, diffractive element systems, which places less of a burden on the optical quality of the laser beam, have also been pursued.

One of the advantage of a DWA is its reduced volume over conventional waterline technology. First, because of the dielectric and switching methods used, the system can generate the required pulse in a single stage of pulse compression. In conventional systems, three to five stages are re- 
quired. Second, the DWA lends itself to a symmetric, evenly electrically stressed geometry. This second advantage allows better integration of the system components and better optimization of the system design. A reduced overall volume results. Further, as the DWA can be fabricated from high permittivity dielectric materials (one to two orders of magnitude greater than those used in conventional machines) higher energy storage densities and low impedances may be achieved. These combined advantages suggest volume reductions of one-to-two order of magnitude can be achieved with DWA technology over conventional water line technology.

Breakdown Characteristics of $\mathrm{TiO}_{2}$ Enect of Dopant Materials

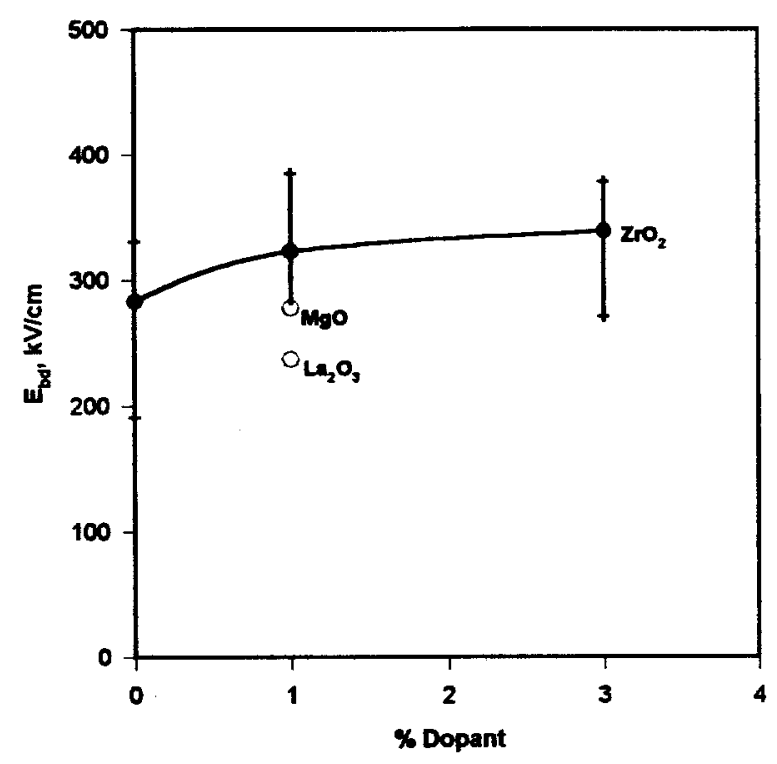

Figure 2 - Breakdown characteristics of $\mathrm{TiO}_{2}$ substrates [5].

In this report, we focused our attention to specific areas of the DWA system. First, we sought to develop the necessary design tools to model and scale the behavior of the high gradient insulator. To perform this task, we concentrated on modeling the discharge processes (i.e., initiation and creation of the surface discharge). In addition, because these high gradient structures exhibit favorable microwave properties in certain accelerator configurations, we performed experiments and calculations to determine the relevant electromagnetic properties. Second, we performed circuit modeling to understand energy coupling to dynamic loads by the asymmetric Blumlein. Further, we have experimentally observed a non-linear coupling effect in certain asymmetric Blumlein configurations. That is, as these structures are stacked into a complete module, the output voltage does not sum linearly and a lower than expected output voltage results. Although we solved this effect experimentally, we performed calculations to understand this effect more fully to allow better optimization of this DWA pulse-forming line system (DWA-PFL). 


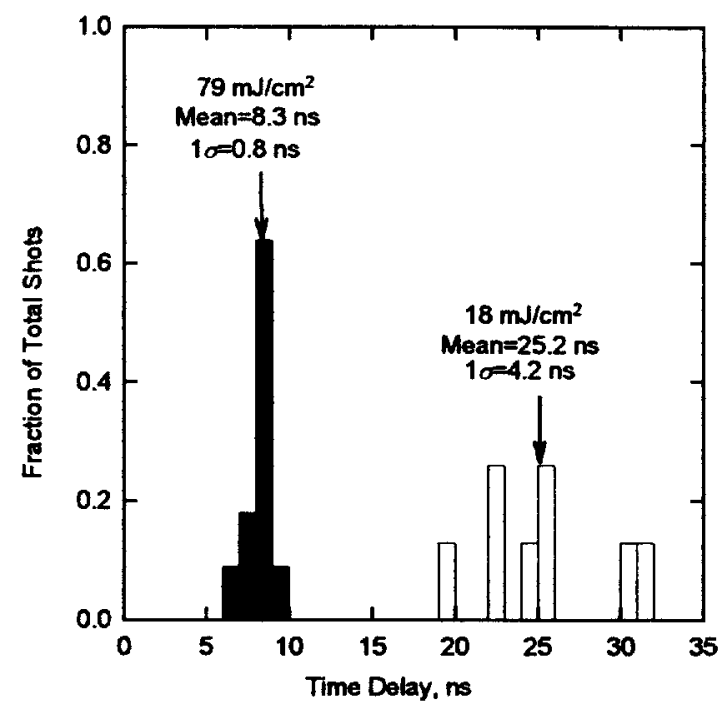

Figure 3 - Jitter Characteristics of the DWA Switches [6].

\section{HIGH GRADIENT INSULATORS}

\section{A. Background}

Over the past several years, we have successfully developed an ultra-high gradient insulator system. These high gradient insulators consists of a series of very thin $(<1 \mathrm{~mm})$ stacked laminations interleaved with conductive planes and exhibit increased breakdown capability (Figure 4).

A certain amount of understanding of the increased breakdown threshold of these structures can be realized from the basic model of surface flashover. The most simplified vacuum surface breakdown model suggests that electrons originating from the cathode-insulator junction are responsible for initiating the failure. When these electrons are intercepted by the insulator, additional electrons, based on the secondary emission coefficient of the surface, are liberated. This effect leaves a net positive charge on the insulator surface, attracting more electrons and leading to escalation of the effect or the so-called secondary emission avalanche breakdown (SEA). It has been shown that full evolution of the discharge occurs within $0.5 \mathrm{~mm}$. Thus, placing slightly protruding metallic structures spaced at an equivalent interval is believed to interrupt the SEA process and allow the insulator to achieve higher gradients before failure.

Alternate explanations include the effects of insulator shielding, interruption of subsurface currents, and equilibration of the induced surface charge. As a result, electron impact on the surface is modified. Or, alternately, by separation of the insulator into $\mathrm{N}-1$ additional decoupled substructures, a local breakdown on the insulator is inhibited from propagating to the remainder of the structure. Never-the-less, our present data shows that these new high gradient insulators can 
sustain an electric field roughly 1.5-4 times the limit of an identical conventional insulator based system (Figure 5) and exhibit similar improvements under more severely stressing bi-polar operation.

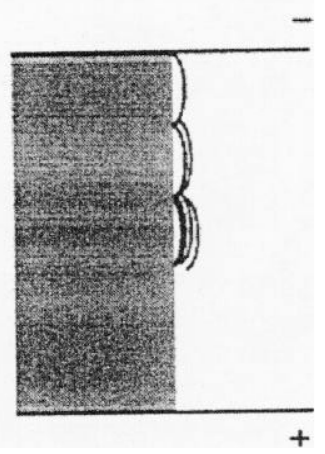

Figure 4 - Conventional (left) and ultra-high gradient insulator (right).

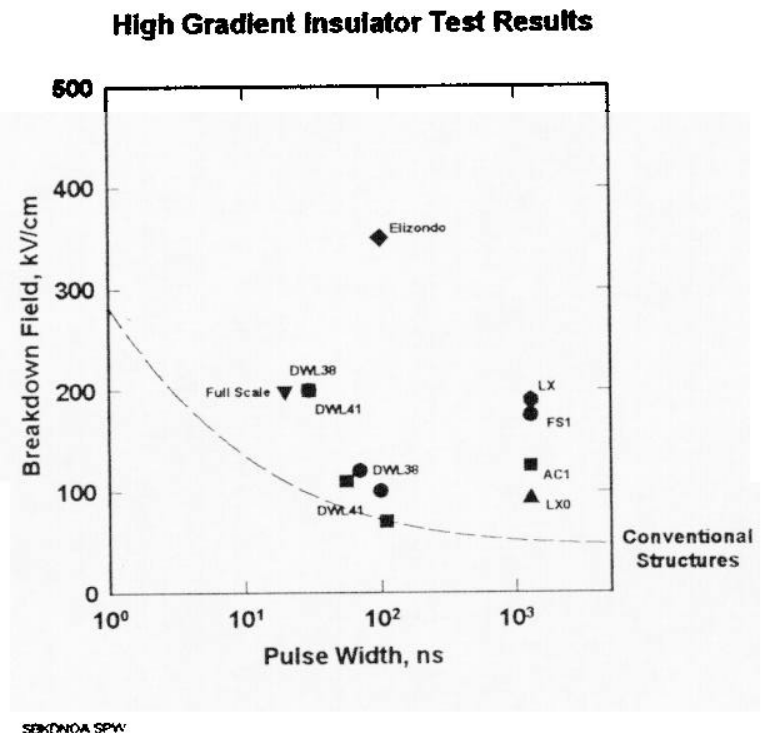

Figure 5 - Performance of various high gradient insulators samples compared to conventional insulator systems.

The high gradient insulator also exhibits microwave properties which suppress or modify microwave modes that are detrimental to beam transport. In present induction accelerators, certain microwave modes, induced by the beam, can develop in the cell cavities. The result is a growing transverse instability which makes the beam difficult to transport. We pursued work which characterized this effect experimentally and computationally. 


\section{B. Insulator Scaling}

Much of the work done to date, although successful, was done in a highly empirical way. This method of optimization, however, is both time consuming and expensive. In this effort we initiated a theoretical effort to explore the scaling of several of the high gradient insulator parameters: dielectric constant, SEA ratio (material surface preparation), thickness and shape of the laminations, etc. In this way, we would be able to easily implement changes to an existing geometry or investigate the properties of the structure further to validate our modeling results.

The basis for our model was the code INDUCT (a hybrid fluids plasma code) which was developed under the Technology Transfer Initiative and has already been used for both glow discharges and filamentary streamers at fixed gas pressures [7]. In order to apply the code to this task, we added modules which computed the rate of evaporation of neutral particles from electrodes and adjust the gas pressure accordingly and the effect of ion/electron impact on the insulator surfaces. This effort was pursued within the general framework of INDUCT, but required the development of appropriate models of the plasma-surface and ion/electron-surface interaction.

The initial focus of this effort was to evaluate present models and develop a better conceptual understanding of the initiation and propagation of high voltage breakdown along the surface of the insulators. Based on this conceptual model a computer model was then pursued to allow detailed simulation analysis.

High voltage breakdown for insulators surrounded by vacuum is believed to take place close to the insulator surface, and not deep within the insulator or far above the insulator in the vacuum. Breakdown occurs on the time scale of nanoseconds, making this a difficult process to study experimentally. The basic physical processes involved are poorly understood and only descriptive models have been proposed. The two most widely accepted published models $[8,9]$ for surface breakdown focus on the initiation process occurring either just below or just above the insulator surface. These models are based respectively on solid state physics phenomena, and on the propagation and emission of electrons through the vacuum just exterior to the insulator surface. Both models lead to surface heating, and evaporation of gas from the insulator. This evaporated gas is the medium where ultimately the voltage breakdown occurs along very localized "streamer" channels.

We have compared processes involved in surface voltage breakdown with other well understood voltage breakdown phenomena. Our conclusion is that an accurate theoretical model of surface breakdown must not only include aspects of both standard models, but must also treat photoionization during the long time scale evolution of the streamer discharge though the evaporated gas [10]. The tip of a propagating streamer is known to produce intense high energy radiation emission which we believe can lead to photo-conduction in the insulator. As conduction in the insulator will strongly modify the voltage which drives the streamer discharge, accurate coupling of the streamer to the insulator must be included. The necessary elements in the model must therefore include: surface physics (electron emission, gas evaporation, surface heating); solid state physics (heating, heat transport, photo-conduction, electrical currents); plasma/gas physics (charged particle and gas transport, ionization, recombination, and chemistry, $u x / x$-ray emission). 
Interactions between all of these processes may be taking place at different times and places during the discharge to determine whether it grows to the point where voltage breakdown actually occurs.

During this first year of this effort, substantial progress was made in the development of a numerical model for surface breakdown based on the existing 2D discharge code INDUCT95. The ability to treat secondary electron emission and electron thermionic/field emission was added. The ion temperature equation was modified to treat high temperatures. A complete re-write was done of the code internal structure setup and numerical bookkeeping scheme to allow for simultaneous modeling of plasma, neutral, and solid state regions. The capability to allow internal metal structures to have a floating potential was added. Static magnetic field effects were added for the electron continuity equations. Atomic data sets were generated for several atomic species including Tungsten. A 1D neutral flow model was developed and tested using an implicit Roe-Reiman solver scheme. Extensive Monte Carlo simulations were conducted for several geometries of secondary electron emission charging of dielectric surfaces.

Examples of results obtained during our first years effort are shown in Figures 6 and 7. This simulation was performed with the updated version of INDUCT95/HYBRID and uses a MonteCarlo treatment of electrons and a fluid treatment of ions to follow the discharge development. Several geometry configurations and surface materials were investigated. The standard configuration studied had an electrode separation of $0.1 \mathrm{~cm}$ with an applied voltage of $50 \mathrm{kV} / \mathrm{cm}$. An insulator with a dielectric constant, $\varepsilon_{\mathrm{r}}=15$ was placed between the electrodes. The dielectric was considered to either have a flat surface (Figure 6) or a step discontinuity (Figure 7) halfway between the electrodes. Electrons were launched with random initial direction from the cathode triple point until they either struck the insulator or the anode. Upon striking the insulator, electrons generated secondary electrons.

The secondary emission yield is a strong function of incident electron energy. Secondary yield profiles were modeled for $\mathrm{Al}_{2} \mathrm{O}_{3}$ and $\mathrm{Cr}_{2} \mathrm{O}_{3}$. The secondary yield for $\mathrm{Al}_{2} \mathrm{O}_{3}$ has a maximum of 2.42 while the $\mathrm{Cr}_{2} \mathrm{O}_{3}$ peaked at 0.98 . A yield greater than unity implies that the electron impact can lead to a positive surface charge on the insulator as more than one electron will be emitted.

Simulation results showed strong positive surface charging for $\mathrm{Al}_{2} \mathrm{O}_{3}$ and weak negative surface charging for $\mathrm{Cr}_{2} \mathrm{O}_{3}$ for the flat dielectric case (Figure 6). Regions of positive surface charge on the insulator attract electrons emitted from the triple point and electrons emitted by secondary emission. This attraction leads to enhanced scattering onto the insulator surface which results in avalanche. For $\mathrm{Cr}_{2} \mathrm{O}_{3}$, the negative surface charge repelled electrons from the triple point, reducing scattering and no avalanche was obtained. As would be expected, the insulator geometry was found to strongly influence surface charging. The step dielectric geometry was modeled for $\mathrm{Al}_{2} \mathrm{O}_{3}$. Strong negative surface charging developed along the step due to high energy electron impact (the secondary emission yield drops below unity at high energy). This negative charge repelled electrons upwards away from the insulator surface, inhibiting the avalanche growth for this geometry. 
Surface Charge

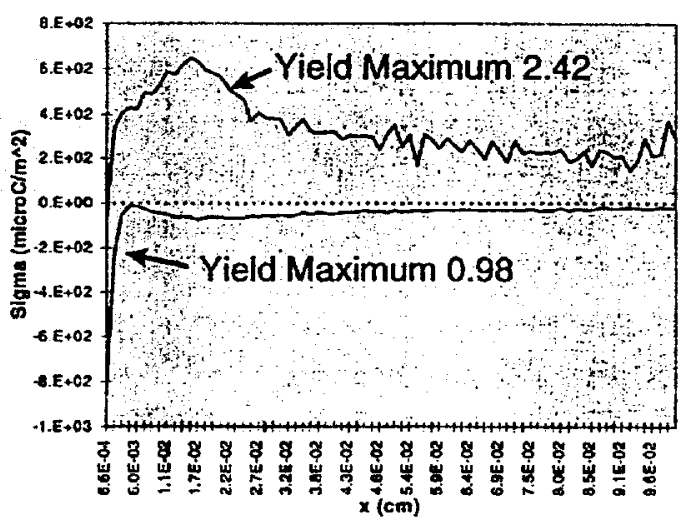

Normal Electric Field

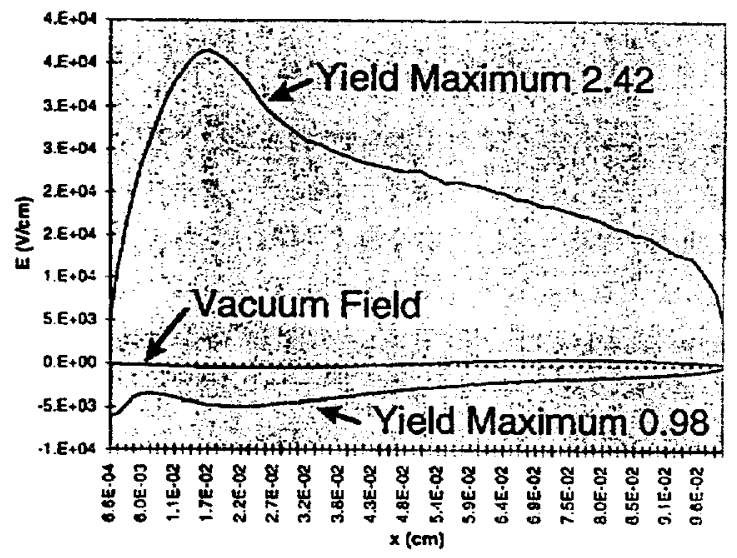

Figure 6 - Typical INDUCT results (straight insulator).

\section{Surface Charge}

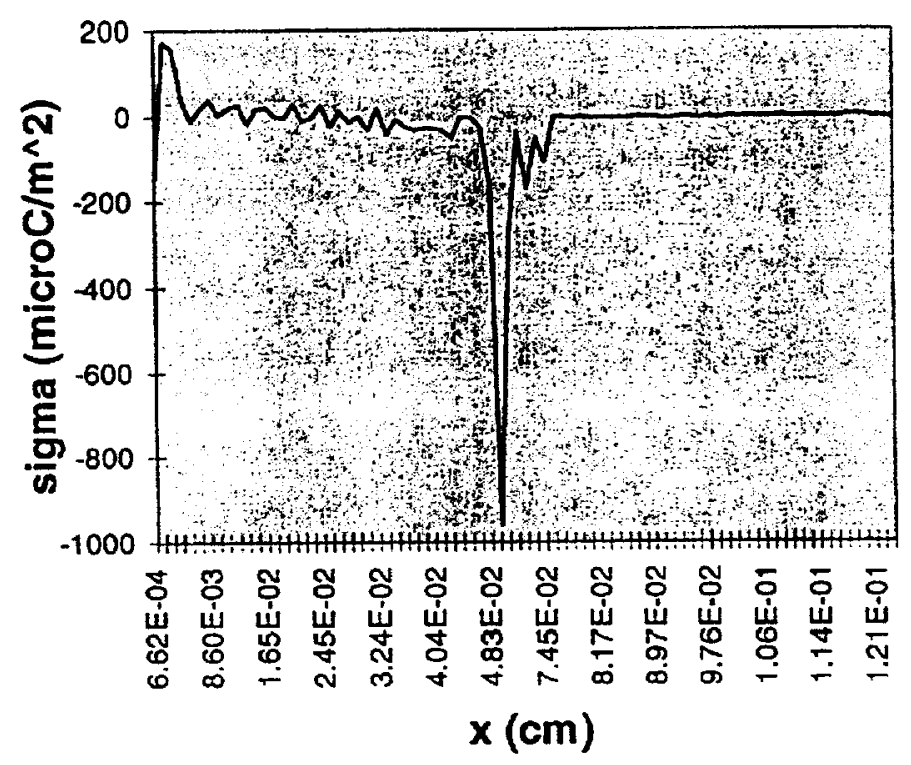

Figure 7 - Typical INDUCT results (step insulator).

\section{High Gradient Insulator Electromagnetic Characteristics}

\section{Introduction.}

Preliminary electromagnetic experimental studies of the High Gradient Insulator (HGI) led to several general observations regarding their characteristics when placed in the opening of a resonant 
cavity, specifically a resonant cavity representative of an induction accelerator module gap $[11,12]$.

(1) The HGI does not create any significant additional resonances in the pill box cavity geometry.

(2) Impedance level for a specific ratio of dielectric to conductor converges rapidly with number of periods.

(3) Ratio of dielectric to conductor is more important than number of periods.

(4) Effects of the HGI are mode dependent, not frequency dependent.

(5) The reduction in peak impedance is roughly proportional to the decrease in dielectric material in the HGI except for the lowest mode for the geometries studied.

(6) Simulations with the AMOS electromagnetic code agreed quantitatively with experimental measurements.

Further a significant issue addressed in this study was the determination of the modes affected by the HGI.

\section{Simulated Cavity, Methodology, and Definitions}

A simple pillbox cavity with a resistive impedance along the outer wall of the cavity was modeled. A relatively large ratio of outer diameter to pipe aperture was used so that multiple resonant modes existed below the cutoff frequency. The basic geometry of the cavity is shown in Figure 8 . The simulated insulator had a $1 \mathrm{~cm}$ radial thickness and was positioned at various radial positions within the cavity. The base HGI design was comprised of five layers of insulator with conducting disks between the insulators. From prior studies, it was felt that this was a sufficient number of layers to model a many layered HGI. The insulator had a dielectric constant of 2.53 and a volume ratio of 4 to 1 with respect to the conducting disks.

Simulations were performed using both the 2.5-D, time-dependent, wakefield code AMOS and the Eigenmode Solver of the MAFIA family of electromagnetic codes. The $\mathrm{TM}_{1 \mathrm{n} 0}$-like resonant modes were studied due their importance in the transverse stability of the charged particle beam in the induction accelerator. $\mathrm{TM}_{1 \mathrm{n} 0}$ refers to the nomenclature used for describing the geometry of the electromagnetic field pattern in a cylindrical resonator. The TM indicates that the magnetic field lines are transverse to the axis and the three subscripts refer to the angular, radial, and longitudinal dependence of the electromagnetic fields. The results should be applicable to all resonant modes.

In earlier work, only AMOS was used for the simulations as it was capable of modeling the ferrite used as a damper in the simulated and actual cavities. The latest release of MAFIA allows for lossy materials with frequency dependent complex permittivities. However, to avoid complications due to different modeling of lossy materials, a pure resistance was chosen for damping in the AMOS simulations and compared to the R/Q generated by the two codes. 


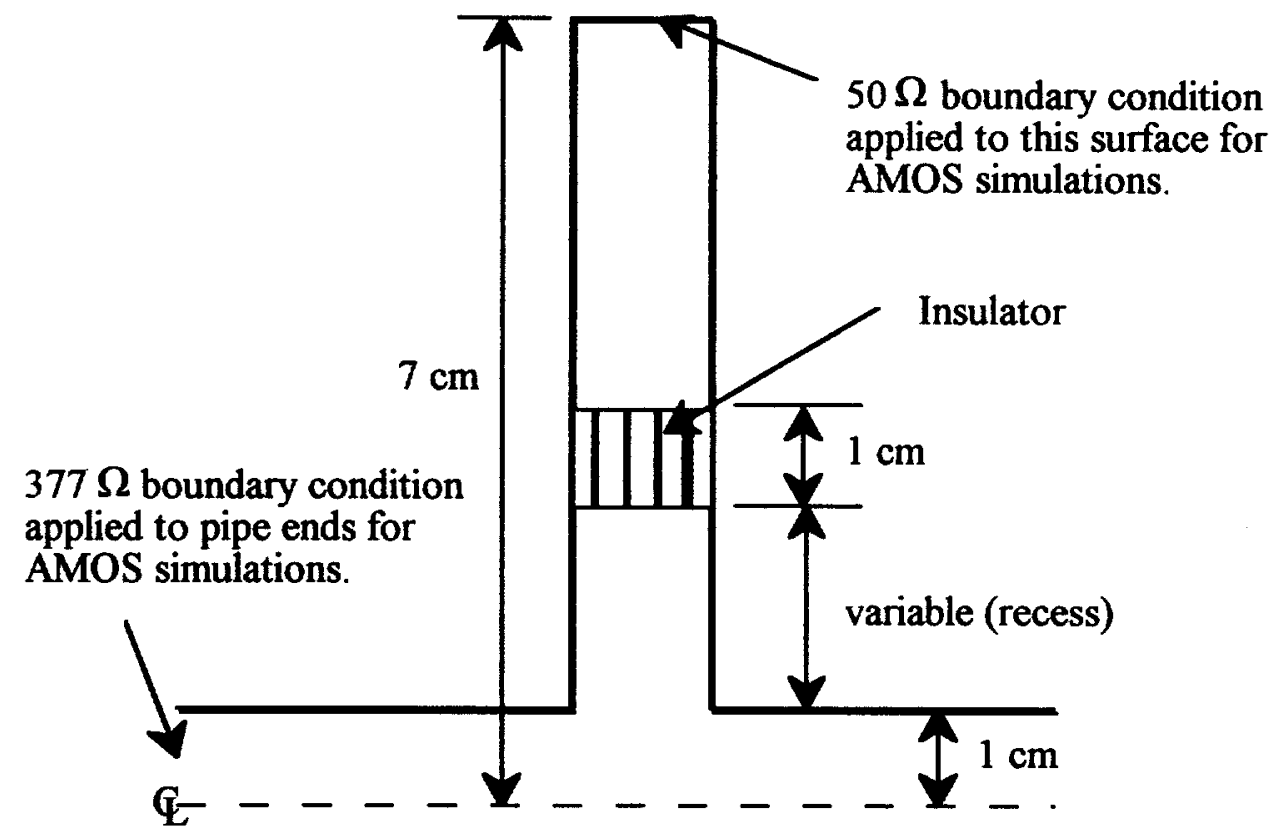

Figure 8. Geometry of simulation model. Cylindrical symmetry.

Some definitions are given for clarification. The quality factor is defined as:

$$
\mathrm{Q}=2 \pi \mathrm{fU} / \mathrm{P} \text { (dimensionless), }
$$

where $\mathrm{f}$ is the resonant frequency, $\mathrm{U}$ is the stored energy, and $\mathrm{P}$ is power dissipation of the mode. An alternative expression for $Q$ used with the impedance spectrum is:

$$
\mathrm{Q}=\mathrm{f} / \Delta \mathrm{f}
$$

where $\Delta f$ is the width of the resonant mode peak at half maximum. Refer to Figure 9 . There are several different impedances that are commonly used. The basic impedance is defined as:

$$
\mathbf{R}=\mathrm{V} 2 / 2 \mathrm{P} \text { (Ohms), }
$$

where $\mathrm{V}$ is the potential change experienced by a charged particle transiting the cavity and includes the transit factor, i.e. it is the integral of the electric field along the particle's path through the cavity assumed to be parallel to the cavity axis. A figure of merit for the design of the cavity comes from the combination of equations (1) and (3):

$$
\mathrm{R} / \mathrm{Q}=\mathrm{V} 2 / 2 \omega \mathrm{U}(\mathrm{Ohms})
$$


The R/Q of the cavity is depends only on the geometry of the cavity and is the quantity calculated by frequency domain (eigenmode solver) codes.

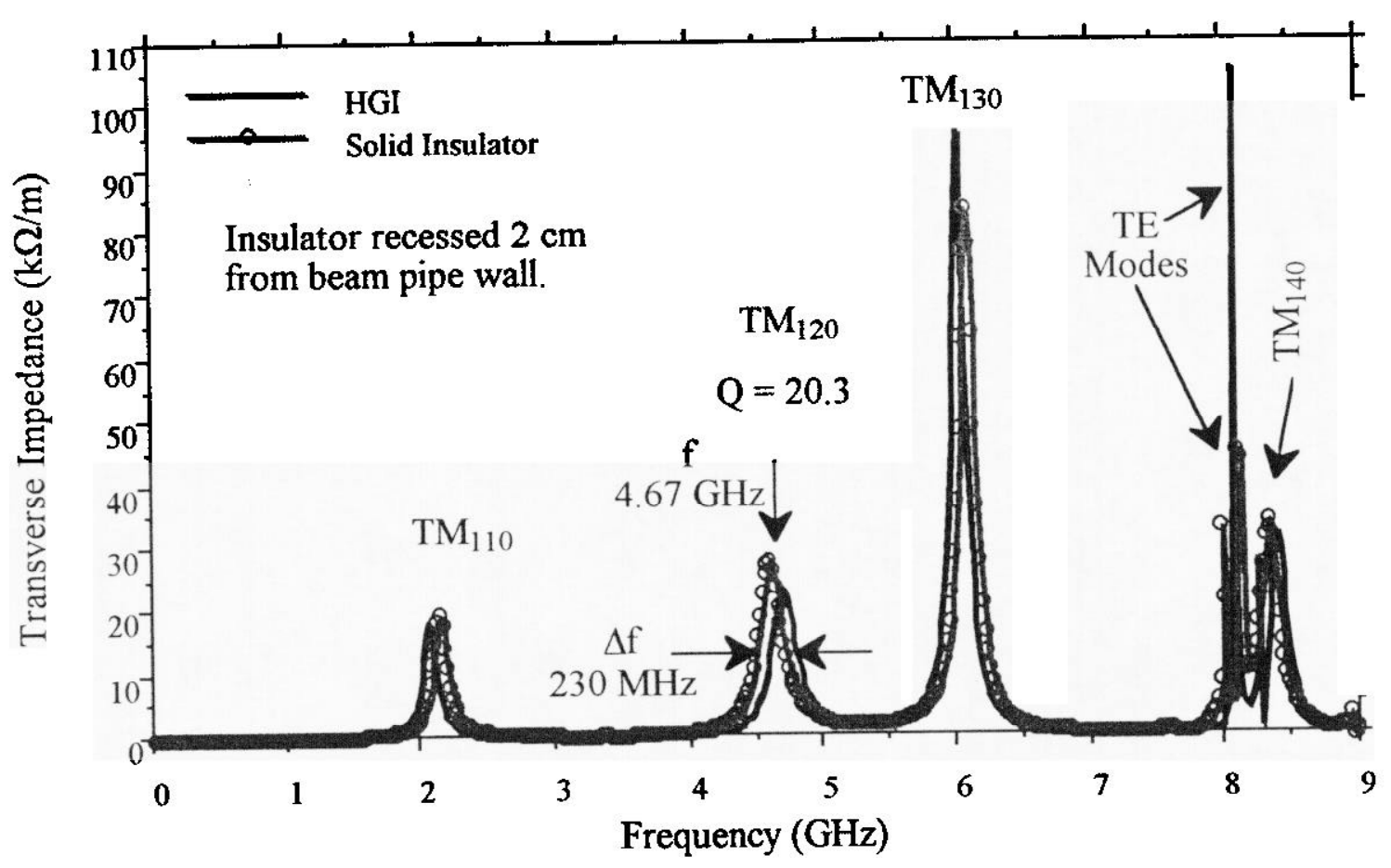

Figure 9 - AMOS simulations results for the pillbox cavity with insulator recessed $2 \mathrm{~cm}$.

For $\mathrm{TM}_{0 \mathrm{n} 0}$-like resonant modes, $\mathrm{V}$ can be considered independent of $\mathrm{r}$, or the radial position of the integration path. For $\mathrm{TM}_{1 \mathrm{n} 0}$-like resonant modes, $\mathrm{V}$ is linear with $\mathrm{r}$ and vanishes on the axis. Thus, the integration is performed at a specific radius, $r_{0}$, and a "normalized" impedance, $R_{N}$ that is independent of radius is used:

$$
\mathrm{R}_{\mathrm{N}} / \mathrm{Q}=\left(\mathrm{c} / \mathrm{r}_{\mathbf{0}} \omega\right)^{2} \mathrm{R} / \mathrm{Q}(\mathrm{Ohms}),
$$

where $\mathrm{c}$ is the speed of light and $\omega$ is $2 \pi \mathrm{f}$.

The figure of merit for beam dynamics is the transverse impedance, $Z \perp$, expresses as:

$$
\mathrm{Z}_{\perp}=(\omega / \mathrm{c})\left(\mathrm{R}_{\mathrm{N}} / \mathrm{Q}\right) \mathrm{Q}_{\text {total }}(\mathrm{Ohms} / \mathrm{m})
$$

where $Q_{\text {total }}$ includes all power dissipation. The transverse instability of the beam increases exponentially with a growth factor proportionally to the transverse impedance and independent of the resonant frequency of the driving resonant mode. AMOS solves the wakefields in the cavity then performs a Fourier transform to calculate $Z \perp$. If the $Q$ of the module gap is large, the transform 
integration must be performed over unrealistically long simulation times. Q's for damped cavities in induction accelerator applications are normally less than 10.

The rationale for performing AMOS simulations was to justify the use of the MAFIA eigenmode solver for studying the RF characteristics of the High Gradient Insulator. AMOS has been shown to simulate experimental results. However, the difficulty with time domain codes are that they are computationally demanding. The frequency domain codes can reduce computational time by as much as two orders of magnitude depending on the problem. AMOS can model more realistic induction modules while the MAFIA eigenmode solver can give much faster insight of the electromagnetic field patterns in a test cavity.

\section{Simulation Results}

Typical results of an AMOS simulation are shown in Figure 9. The insulator was recessed $2 \mathrm{~cm}$ from the beam pipe wall. Results for both a solid insulator and the basic HGI design are shown. Table 1 lists a comparison of pertinent parameters calculated analytically for a cylindrical resonator (no insulator), generated by the eigenmode solver, and determined from the AMOS simulation.

Table 1. Comparison of Simulation Results

\begin{tabular}{|l|c|c|c|c|c|}
\hline \multicolumn{1}{|c|}{ METHOD } & PARAMETER & $\mathrm{TM}_{110}$ & $\mathrm{TM}_{120}$ & $\mathrm{TM}_{130}$ & $\mathrm{TM}_{140}$ \\
\hline Resonator & $\mathrm{f}(\mathrm{GHz})$ & 2.29 & 4.19 & 6.07 & 7.95 \\
\hline AMOS & & & & & \\
\hline Solid Insulator & $\mathrm{f}(\mathrm{GHz})$ & 2.13 & 4.58 & 6.07 & 8.32 \\
\hline & $\mathrm{Z}_{\perp} / \mathrm{Q}(\mathrm{k} \Omega / \mathrm{m})$ & 1.29 & 1.30 & 1.93 & 0.68 \\
\hline HGI & $\mathrm{f}(\mathrm{GHz})$ & 2.04 & 4.67 & 6.01 & 8.38 \\
\hline & $\mathrm{Z}_{\perp} / \mathrm{Q}(\Omega / \mathrm{m})$ & 1.20 & 1.18 & 2.08 & 0.56 \\
\hline Eigenmode & & & & & \\
\hline Solid Insulator & $\mathrm{f}(\mathrm{GHz})$ & 2.12 & 4.56 & 6.05 & 8.30 \\
\hline & $\mathrm{Z}_{\perp} / \mathrm{Q}(\Omega / \mathrm{m})$ & 1.19 & 1.25 & 1.85 & 0.68 \\
\hline HGI & $\mathrm{f}(\mathrm{GHz})$ & 2.04 & 4.67 & 6.02 & 8.36 \\
\hline & $\mathrm{Z}_{\perp} / \mathrm{Q}(\Omega / \mathrm{m})$ & 1.13 & 1.12 & 1.95 & 0.59 \\
\hline
\end{tabular}

Several comments should be made about Figure 9 and results shown in Table 1. The cutoff frequency for the beam pipe is $8.8 \mathrm{GHz}$ for the $\mathrm{TE}_{11}$ and $11.5 \mathrm{GHz}$ for the $\mathrm{TM}_{01}$ waveguide modes. Above $10 \mathrm{GHz}$ there were no significant resonances found by AMOS and the eigenmode results were not reliable due to the effect of boundary conditions. The effect of the pipe aperture on the 
cylindrical resonator modes becomes more pronounce for the higher frequency modes (particularly those near the cutoff frequencies). The four trapped (below cutoff) $\mathrm{TM}_{\mathrm{In} 0}$ modes were well modeled by both codes. Near $8.0 \mathrm{GHz}$, AMOS generated several very high $\mathrm{Q}$, very low $Z_{\perp} / Q$ resonances that were not well resolved due to the high $Q$. These resonances were also found in the eigenmode solutions by imposing a magnetic boundary condition along the plane perpendicular to the axis and at the center of the cavity. These are most likely TE modes that do not significantly couple to the beam, i.e. $R / Q \sim 0$. They are noticeable in the AMOS simulations because the electromagnetic field patterns do not extend sufficiently into the simulated cavity to be damped. Therefore, $Q \sim$ infinity. If finite wall conductivities were included in the simulation, these modes would be negligible. A new result not seen in previous work is that the HGI can increase $Z_{\perp} / Q$ (see $T M_{130}$ column of Table 1 ).

AMOS results were checked against the eigenmode solver for approximately seven different radial locations and/or configurations of the HGI design with excellent agreement for frequency and $Z_{\perp} / Q$. For the final part of the study only eigenmode solver results are presented.

The close agreement between AMOS and the eigenmode solver indicates that the electromagnetic field patterns are similar. This should be expected because of the orientation of the conducting disks. For the $\mathrm{TM}_{\mathrm{ln} 0}$-like modes, the disks are perpendicular to the electric field lines and parallel to the magnetic field lines. This orientation will minimize the perturbation to the fields and also explains the relatively fast convergence of the impedance as the number of layers in the HGI is increased while holding the ratio of insulator to conductor constant. Once the disks are sufficiently thin, perturbation theory can be used to show that variation to the cavity's $R / Q$ is only dependent on the total volume of the disks.

Table 2 lists $\mathbf{R}_{\mathbf{N}} / \mathrm{Q}$ 's for different radial positions of a solid insulator and a HGI for the first three $T_{M} M_{1 n}$ modes. The $R_{N} / Q$ for the pillbox cavity without insulator is given for comparison. The structure behind the impedances shown in Table 2 can be seen in plots of the electric field energy density as a function of radial distance. See Figures 10 through 15 . The energy density has been normalized by multiplying by the radius and dividing by the total energy. If this normalized energy is integrated over $r, z$, and $\theta$, the result will be one. The field density of the pillbox without insulator is shown for reference.

The two radial walls of the cavity can be thought of as parallel plates of a capacitor. The insulator with its dielectric constant $>1$ will have a greater energy density than the case with no insulator. The HGI simply increases the capacitance of the insulator by decreasing the effective distance between the walls. By comparing the various plots with the respective $R_{N} / Q$ 's in Table 2, one can deduce that, when the insulator is located so that the energy density is shifted towards (away from) the centerline, the $R_{N} / Q$ will be increased (decreased) over the no insulator case. The HGI simply enhances this effect. Thus as a general rule, if inserting a solid insulator will increase the $R_{N} / Q$, an $H G I$ will further increase the $R_{N} / Q$. Similarly, if inserting a solid insulator will decrease the $R_{N} / Q$, an $H G I$ will further decrease the $R_{N} / Q$. 
Table 2. $R_{N} / Q(O h m s)$ for different modes and insulator configuration.

\begin{tabular}{|l|l|l|l|l|l|l|l|l|l|}
\cline { 2 - 10 } \multicolumn{1}{c|}{} & \multicolumn{3}{c|}{ TM $_{110}$} & \multicolumn{3}{c|}{ TM $_{120}$} & \multicolumn{3}{c|}{ TM$_{130}$} \\
\hline recess & None & Solid & HGI & None & Solid & HGI & None & Solid & HGI \\
\hline flush & 25.0 & 45.7 & 47.9 & 17.8 & 29.3 & 26.9 & 9.72 & 6.66 & 4.82 \\
\hline $0.5 \mathrm{~cm}$ & 25.0 & 41.8 & 44.9 & 17.8 & 15.8 & 13.0 & 9.72 & 5.52 & 4.16 \\
\hline $1.0 \mathrm{~cm}$ & 25.0 & 36.7 & 38.4 & 17.8 & 10.4 & 7.86 & 9.72 & 9.19 & 8.61 \\
\hline $1.5 \mathrm{~cm}$ & 25.0 & 31.4 & 32.0 & 17.8 & 9.37 & 7.06 & 9.72 & 15.3 & 16.8 \\
\hline $2.0 \mathrm{~cm}$ & 25.0 & 26.9 & 26.4 & 17.8 & 13.1 & 11.5 & 9.72 & 14.6 & 15.5 \\
\hline $2.5 \mathrm{~cm}$ & 25.0 & 23.2 & 22.2 & 17.8 & 20.1 & 21.5 & 9.72 & 8.67 & 7.67 \\
\hline $3.0 \mathrm{~cm}$ & 25.0 & 20.3 & 18.9 & 17.8 & 23.3 & 25.0 & 9.72 & 7.03 & 5.90 \\
\hline
\end{tabular}

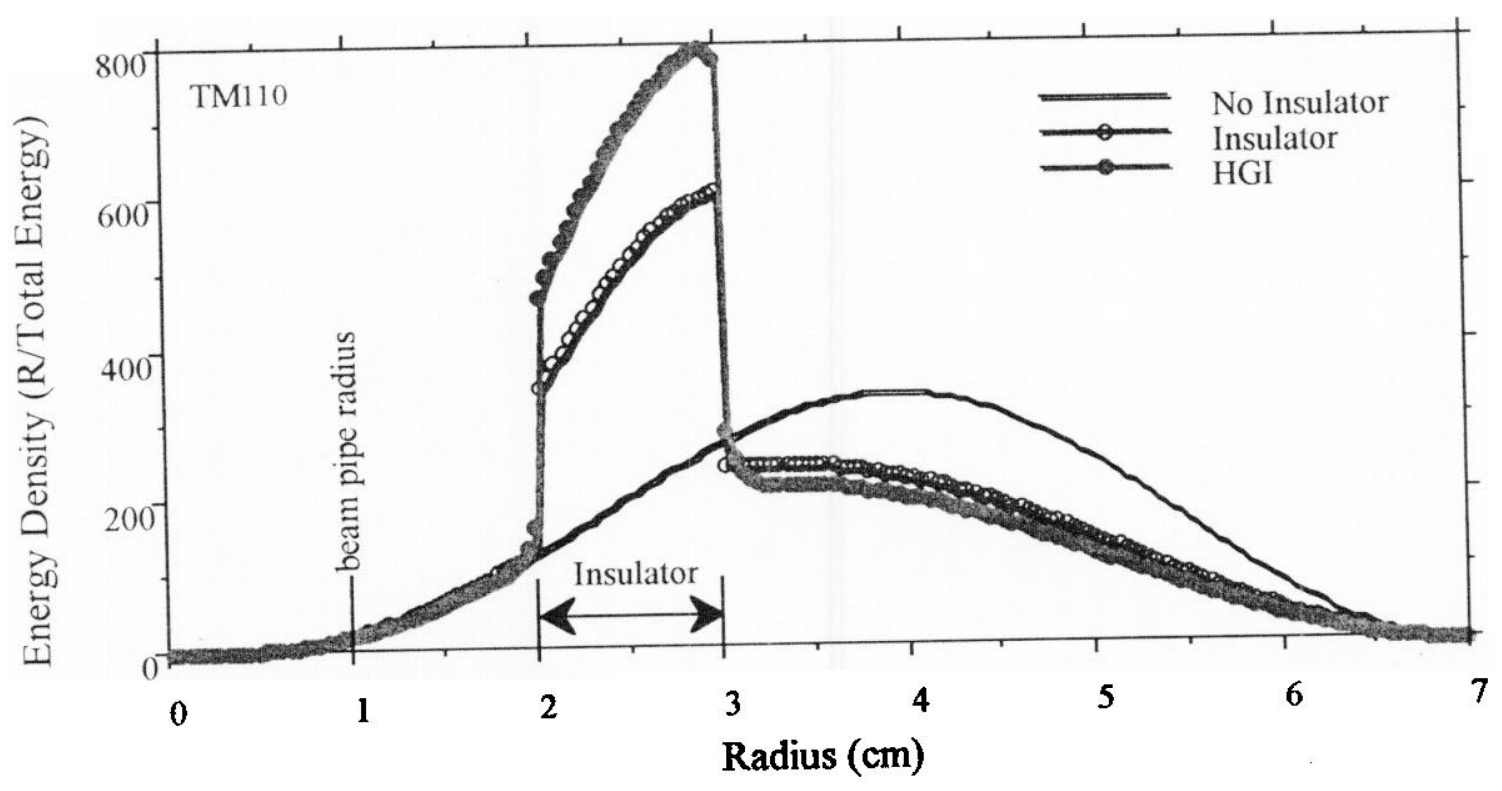

Figure 10 - Normalized energy density (electric field) for the $\mathrm{TM}_{110}$ mode is plotted as a function of radius. The insulator is recessed $1 \mathrm{~cm}$ from the pipe wall. The shift in energy density towards the centerline for the insulators indicates an increase in $\mathrm{R} / \mathrm{Q}$. 


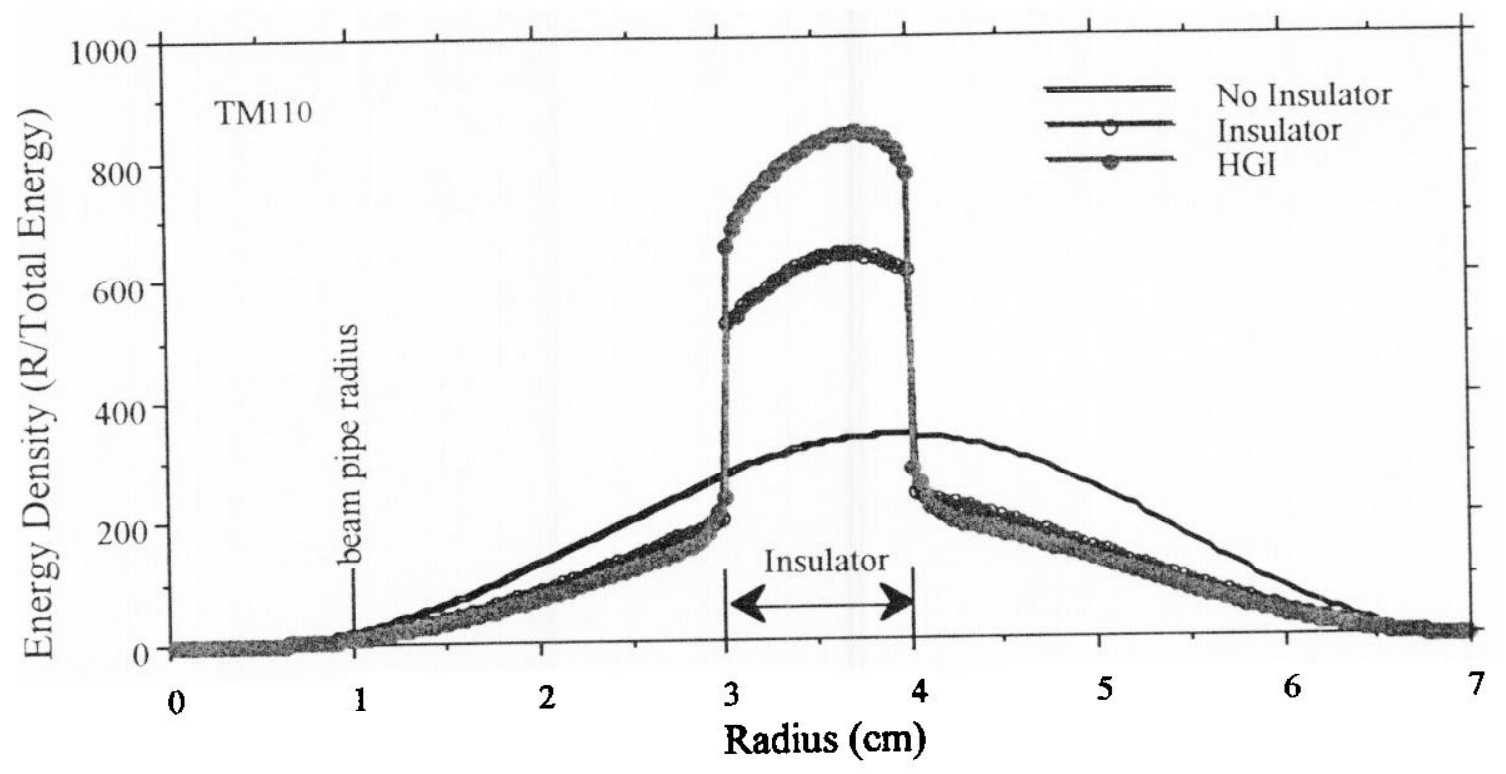

Figure 11 - Normalized energy density (electric field) for the $\mathrm{TM}_{110}$ mode is plotted as a function of radius. The insulator is recessed $2 \mathrm{~cm}$ from the pipe wall. The shift in energy density away from the centerline for the insulators indicates a decrease in $R / Q$.

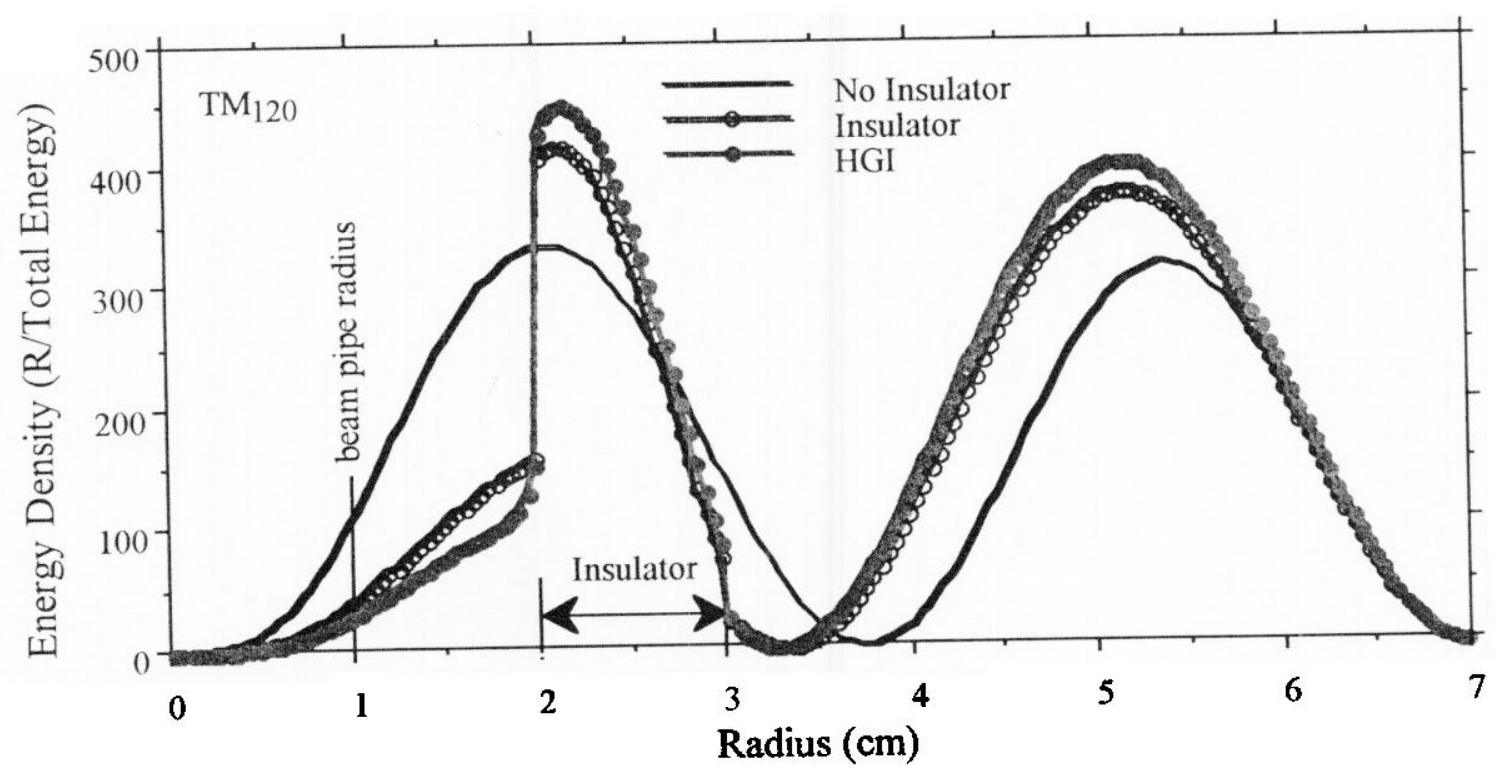

Figure 12 - Normalized energy density (electric field) for the $\mathrm{TM}_{120}$ mode is plotted as a function of radius. The insulator is recessed $1 \mathrm{~cm}$ from the pipe wall. The shift in energy density away from the centerline for the insulators indicates a decrease in $R / Q$. 


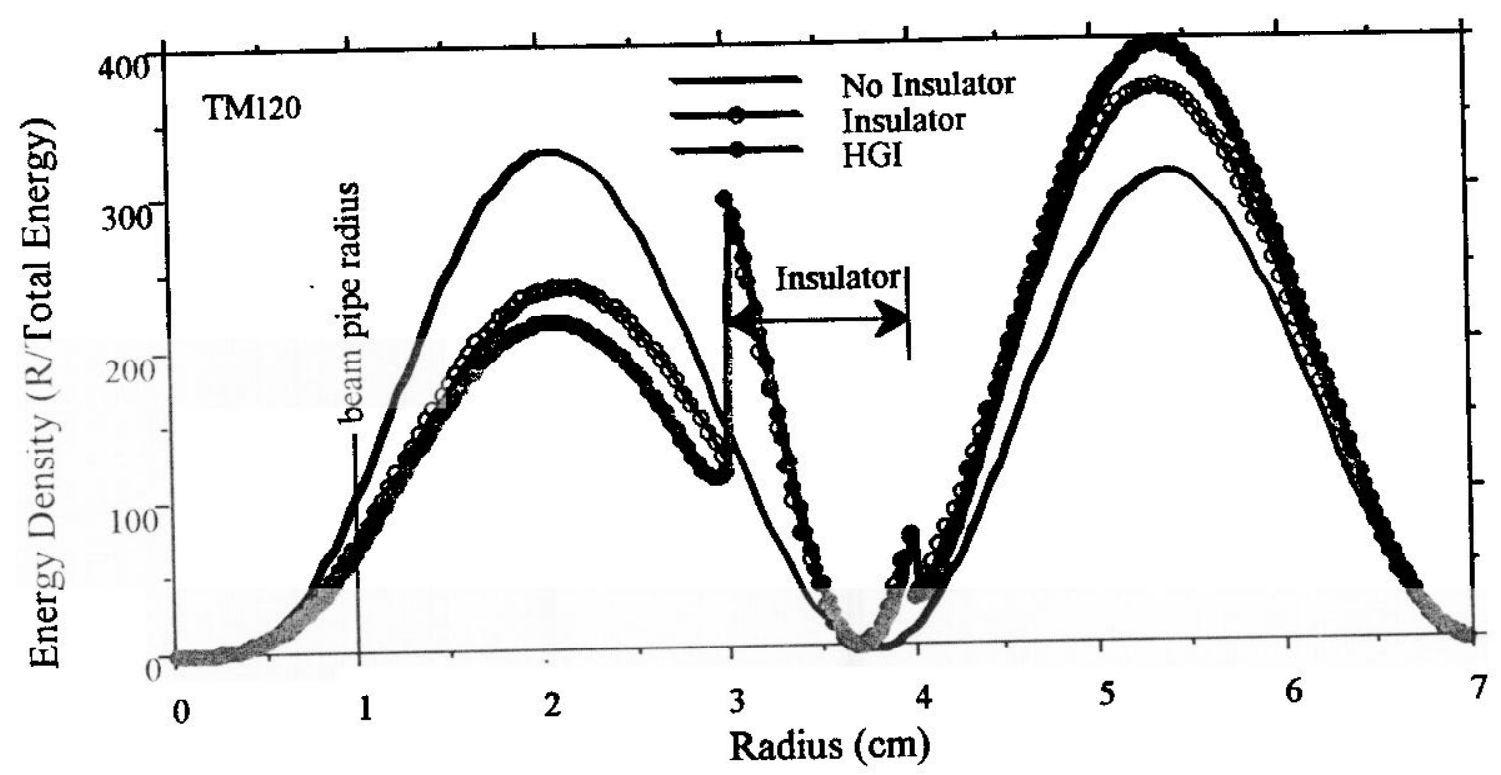

Figure 13 - Normalized energy density (electric field) for the $\mathrm{TM}_{120}$ mode is plotted as a function of radius. The insulator is recessed $2 \mathrm{~cm}$ from the pipe wall. The shift in energy density away from the centerline for the insulators indicates a decrease in $R / Q$.

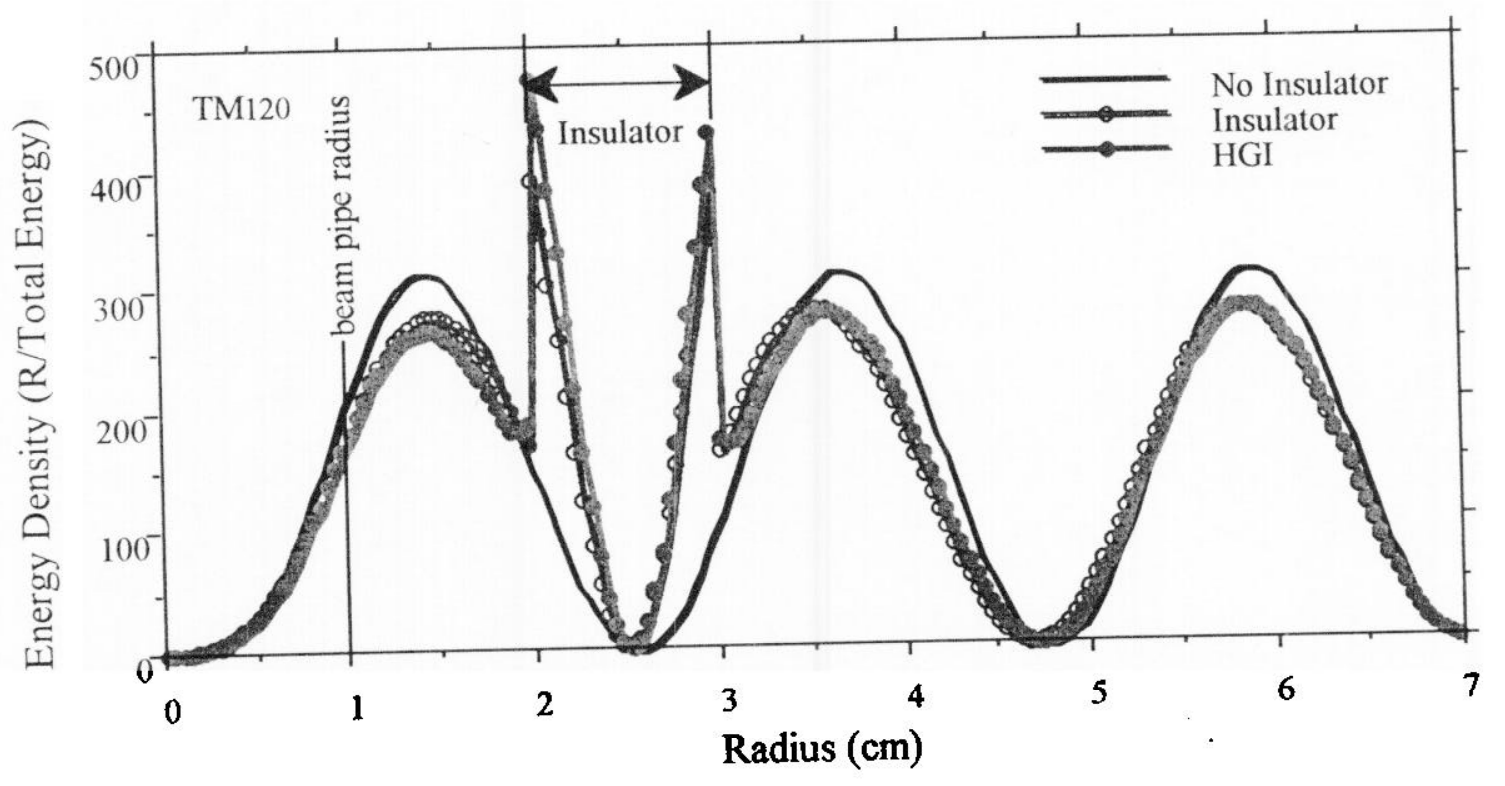

Figure 14 - Normalized energy density (electric field) for the $\mathrm{TM}_{130}$ mode is plotted as a function of radius. The insulator is recessed $1 \mathrm{~cm}$ from the pipe wall. The shift in energy density away from the centerline for the insulators indicates a decrease in $R / Q$. 


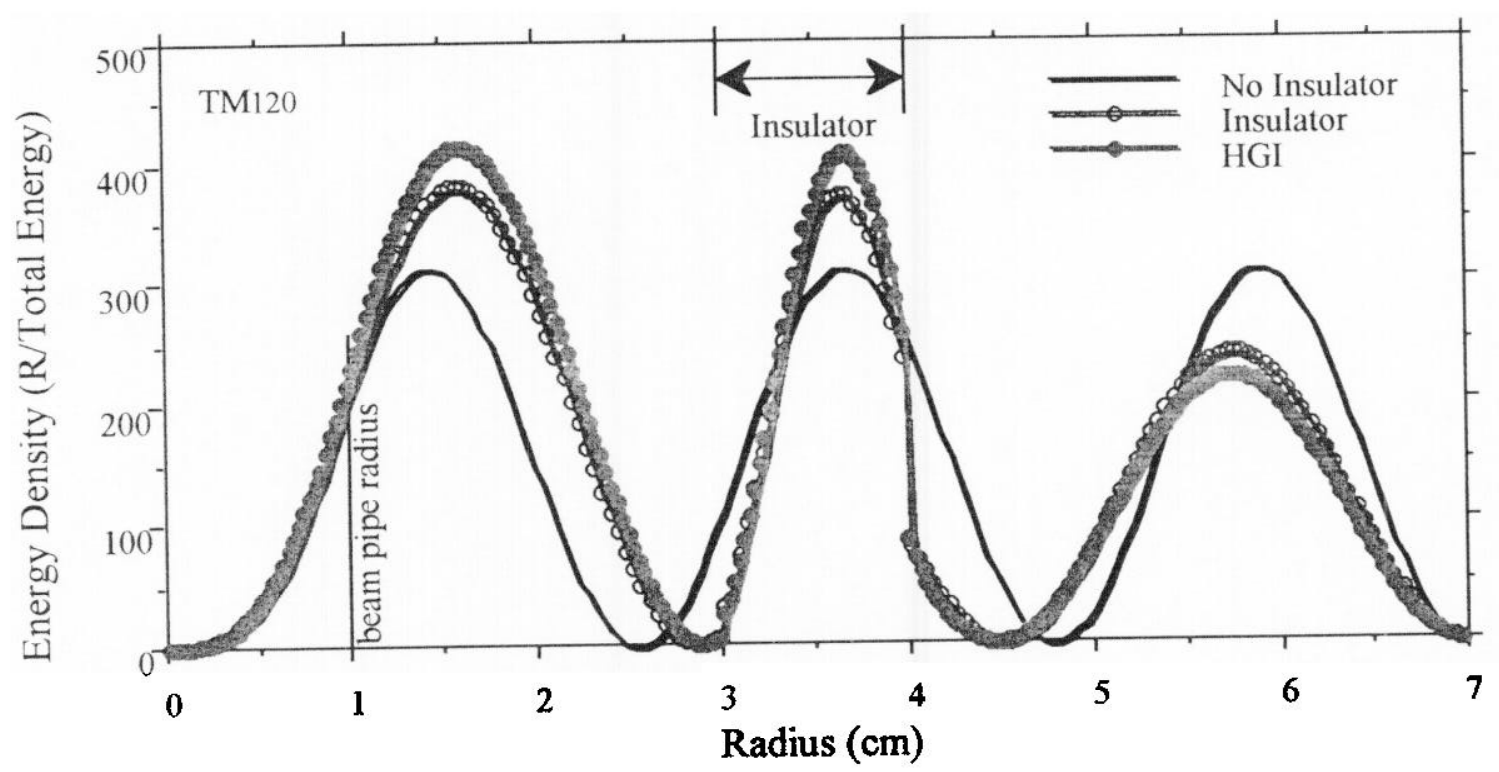

Figure 15 - Normalized energy density (electric field) for the $\mathrm{TM}_{130}$ mode is plotted as a function of radius. The insulator is recessed $2 \mathrm{~cm}$ from the pipe wall. The shift in energy density towards the centerline for the insulators indicates an increase in $\mathrm{R} / \mathrm{Q}$.

\section{Conclusions}

The electromagnetic characteristics of the HGI are due to perturbing the geometry of the resonant cavity. Careful incorporation of HGI's into an induction cell design should always lead to much lower impedance than that of a conventional solid insulator. However, non-desirable effects such as additional resonant modes and increased impedance can result. Important aspects of the HGI to the cell gap design are:

(1) The higher voltage hold off of the HGI allow for narrower gaps. The transverse impedance scales directly with gap width, so a factor of two reduction is expected.

(2) Proper location of the HGI radially in the gap can lower the R/Q by another factor of two. This is particularly true of the higher frequency modes that are normally the most difficult to reduce in conventional designs. If further testing shows that the insulator to conductor ratio of the HGI can be further lowered while maintaining the voltage hold off, the R/Q can be further reduced.

(3) Proper location of the HGI and absorbers can significantly improve cell damping, i.e. lower cell $Q$, which means even lower effective impedance.

(4) These effects can be adequately simulated by present computer models 


\section{DIELECTRIC WALL ACCELERATOR PULSE-FORMING LINE}

\section{A. Background}

The Dielectric Wall Accelerator (DWA) Pulse-forming Line (PFL) is made of a series of asymmetric Blumleins. Each Blumlein consists of two transmission lines with different dielectric constants. It can provide high field gradient and is capable of providing a fast electrical pulse in a single step process. It has the advantage of being more compact than the conventional technology.

In our initial studies of the DWA, we empirically discovered that for more complex configurations used to extend pulsewidth (where the transmission line is slotted with a spiral to lengthen the path of the propagating wavefront) that a certain amount of pulse energy from an adjoining line can adversely couple to the next nearest neighboring Blumleins. The resultant effect is a lack of linear scaling of the output pulse to the number of modules in the system. However, elimination of the spiral slots would eliminate this problem but would increase the system volume. Thus, there is an optimum slot width which will maintain the required pulsewidth but will minimize this interstage coupling.

In this portion of the study, we analyzed and modeled the propagation characteristics of the Blumleins in DWA-PFL using transient analysis and Micro-Cap software. Output efficiency of a Blumlein which is the ratio of the output energy over the input energy was obtained for various geometries and dielectric constants of the transmission lines, and different loads at the output which include both constant and dynamic loads. Maximum output efficiency was obtained by optimizing the geometry and dielectric constants of a Blumlein for a given output load. Since cross coupling between transmission lines in DWA due to the fringing fields around the structure can have a significant effect on the output performance, we have used a 2-D finite element code to model the TEM coupling of transmission lines for the DWA.

\section{B. Analysis and Simulation of the Blumlein Structure}

The propagation characteristics and output of a Blumlein in DWA-PFL have been analyzed and simulated using transient analysis and Micro-Cap software. The two transmission lines in each Blumlein are composed of different dielectric materials. The one with lower dielectric constant $\left(\varepsilon_{\mathrm{f}}\right)$ is referred to as the fast line, and the other one $\left(\varepsilon_{\mathrm{s}}\right)$ as the slow line. Initially, both lines in each Blumlein are charged to $V_{0}$. Because the two lines have opposite polarities, the net voltage is zero. After a short circuit is applied to one end of the Blumlein, wave in the fast line starts propagating at a velocity:

$$
v_{f}=\frac{c}{\sqrt{\varepsilon_{f}}}
$$

and the wave in the slow line propagates at a velocity:

$$
v_{s}=\frac{c}{\sqrt{\varepsilon_{s}}}
$$


where $c$ is the speed of light. For a Blumlein of length $L$ with an open circuit load, at time $t=L / v_{f}$ after the short circuit is applied $(t=0)$, the polarity on the other end of the fast line is reversed. This results in a net voltage of $2 V_{0}$ across the output of the asymmetric Blumlein. For $\varepsilon_{3}<9 \varepsilon_{f}$, this voltage will persist until $t=L / v_{\mathfrak{t}}$. For $\varepsilon_{\mathrm{s}}>9 \varepsilon_{\mathrm{f}}$, the net voltage will go back to 0 at $\mathrm{t}=3 \mathrm{~L} / \mathrm{v}_{\mathrm{f}}$ before reaching $t=L / v_{\mathbf{s}}$. Since DWA-PFL consists of a stack of asymmetric Blumleins, a high voltage output can be generated.

The voltage and current on the two transmission lines can be found by solving the transmission line equations:

and:

$$
\frac{\partial V}{\partial z}=-L \frac{\partial I}{\partial t}
$$

$$
\frac{\partial I}{\partial z}=-C \frac{\partial V}{\partial t}
$$

where $\mathrm{L}$ and $\mathrm{C}$ are the inductance and capacitance per unit length respectively. The characteristic impedance of the line is:

$$
Z_{0}=\sqrt{\frac{L}{C}}
$$

The structure described can be illustrated by simulations of the circuit shown in Figure 16.

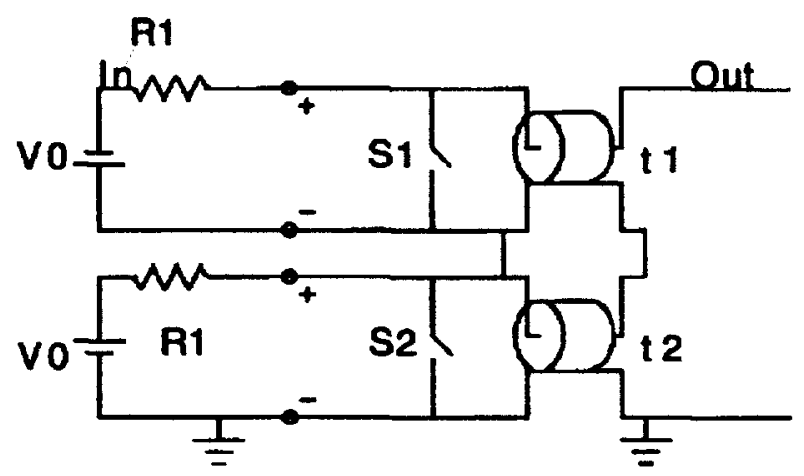

Figure 16. - Configuration of a Blumlein structure terminated with an open load.

It consists of two transmission lines $t 1$ and $t 2$ with length $L=0.45 \mathrm{~m}$. The dielectric constants of the materials inside $\mathrm{t} l$ and $\mathrm{t} 2$ are $\varepsilon_{\mathrm{f}}=10.8$ and $\varepsilon_{\mathrm{a}}=85$, respectively. Therefore, $\mathrm{t} 1$ is the fast line 
and $t 2$ is the slow line. The characteristic impedance of the lines $Z_{0}$ is 50 ohms. Both lines are initially charged to $V_{0}=10 \mathrm{~V}$ with opposite polarities. At $t=10 \mathrm{~ns}$, the switches $S 1$ and $S 2$ are closed and the inputs become short-circuited. Figure 17 shows the voltage output from the configuration shown in Figure 16.

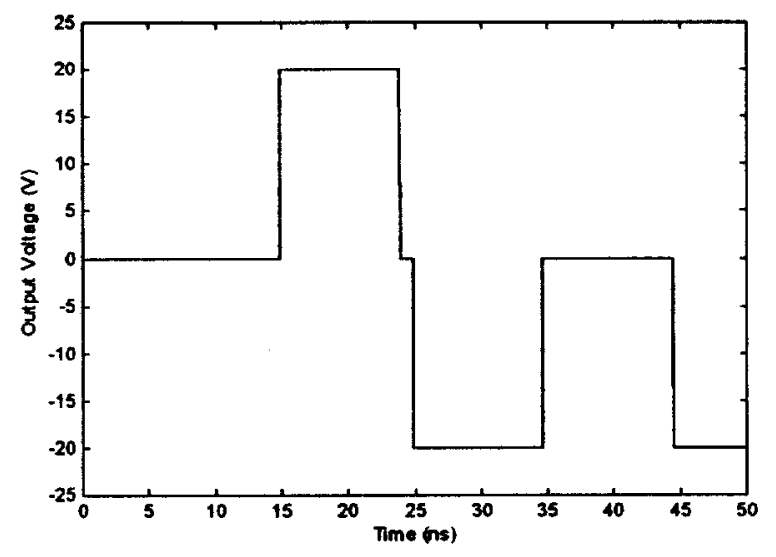

Figure 17 - Transient response of a Blumlein structure terminated with an open load.

The output is consistent with the prediction using the transient analysis. At $t=10 \mathrm{~ns}+\mathrm{L} / \mathrm{v}_{\mathrm{f}}=$ $14.93 \mathrm{~ns}$, the net output voltage is $2 \mathrm{~V}_{0}=20 \mathrm{~V}$. Since $\varepsilon_{3}<9 \varepsilon_{\mathrm{t}}$, the net voltage goes back to zero at $t=10 \mathrm{~ns}+\mathrm{L} / \mathrm{v}_{\mathrm{s}}=23.85 \mathrm{~ns}$. The length of the pulse at the output of the structure depends on the length of the transmission lines $\mathrm{L}$ and the materials inside the transmission lines.

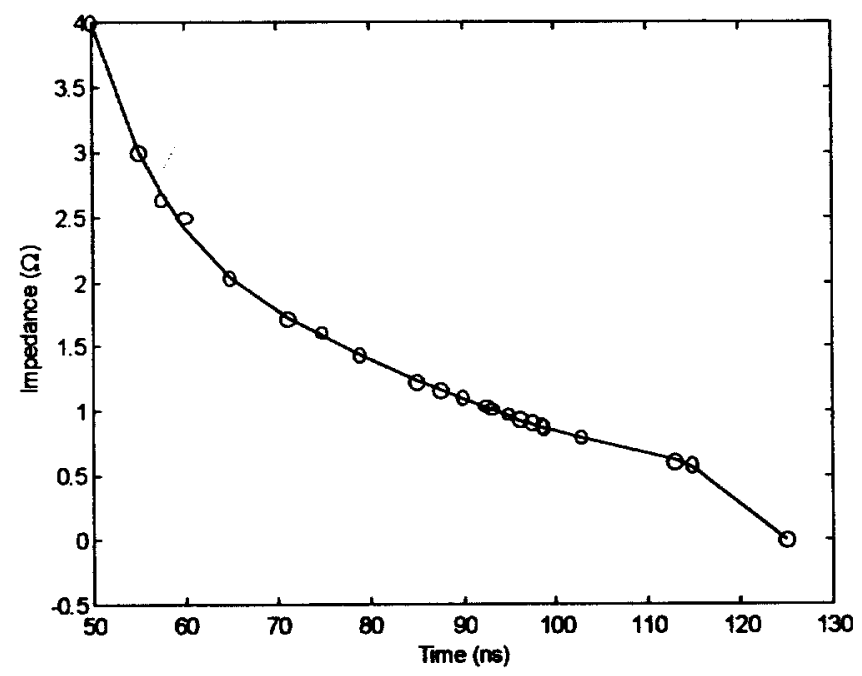

Figure 18 - Impedance of a plasma load versus time after the open-circuit phase. The experimental data are represented by "o". The solid line is obtained using a polynomial fit from the data. 
For our applications, the types of output load for the structure include a plasma whose impedance varies as a function of time. Therefore, it's useful to simulate and examine the output of a Blumlein with a plasma load. To describe the impedance of the plasma load when it starts decreasing, we use a polynomial fit to find the equation from experimental data. The impedance curve of the plasma load to be used in the simulation circuit after the open-circuit phase is shown in Figure 18. We assume the load impedance drops from a very high value to $4 \Omega$ at $t=50 \mathrm{~ns}$.

The configuration of a Blumlein structure terminated with a plasma load is shown in Figure 19. It consists of two transmission lines: $t 1$ with dielectric constant $\varepsilon_{\mathrm{f}}=10.8$, and $\mathrm{t} 2$ with dielectric constant $\varepsilon_{\mathrm{s}}=95$. The length $\mathrm{L}$ and characteristic impedance $Z_{0}$ of the lines are $0.45 \mathrm{~m}$ and $0.82 \Omega$, respectively. The output load is modeled as a switch $S 3$ in series with a resistor $R 2$ with its resistance varies as a function of time. The values of $R 2$ is obtained from the impedance curve in Figure 18. Both lines are initially charged to $V_{0}$ (in our case, $1 \mathrm{MV}$ ) with opposite polarities. At $t=22.85 \mathrm{~ns}$, the switches are closed and the inputs become short-circuited. Before $t=50 \mathrm{~ns}$, the plasma load is an open circuit load. At $t=50 \mathrm{~ns}$, switch $\mathrm{S} 3$ is closed and the load resistance drops to $4 \Omega$.

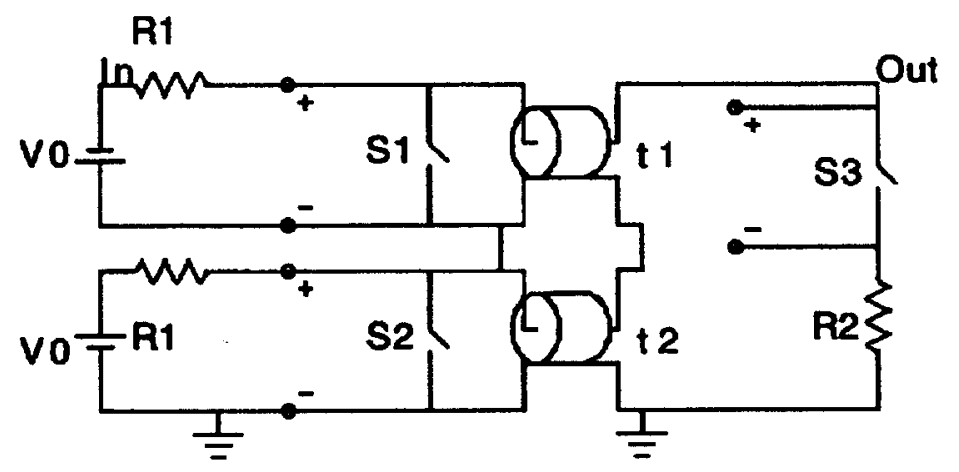

Figure 19 - Configuration of a Blumlein structure terminated with a plasma load.

Figure 20 shows the plot of output voltage of a Blumlein with a plasma load versus time. At $t$ $=22.85 \mathrm{~ns}+\mathrm{L} / \mathrm{v}_{\mathrm{f}}=47.5 \mathrm{~ns}$, the net output voltage is $2 \mathrm{~V}_{0}=2 \mathrm{MV}$ which is what we expected from an open circuit load. When the load impedance decreases, the net output voltage also decreases. The output voltage goes to zero at $\mathrm{t}=22.85 \mathrm{~ns}+\mathrm{L} / \mathrm{v}_{\mathrm{s}}=96 \mathrm{~ns}$.

In addition to the output voltage, output efficiency is another factor which needs to be considered during the design process. Output efficiency is the ratio of the output energy over the input energy. In our study, output efficiencies were obtained for various geometries and dielectric constants of the transmission lines, and different loads at the output. Maximum output efficiency was obtained by optimizing the geometry and dielectric constants of the transmission lines for a given output load. Figures 21-23 show some of the simulation results for a Blumlein terminated with a plasma load. 


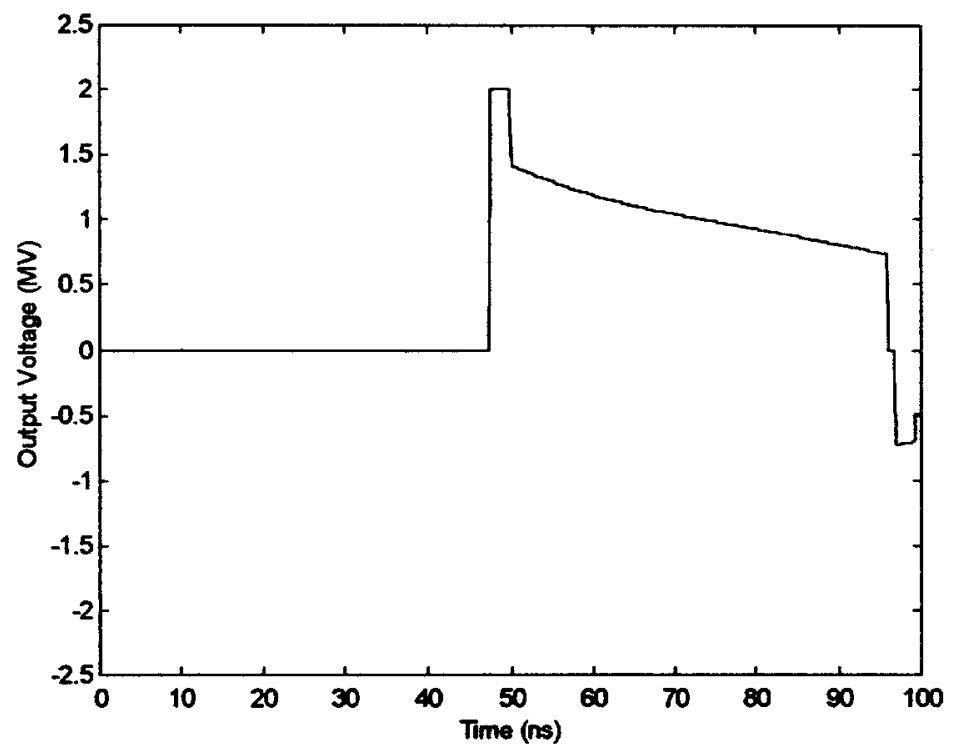

Figure 20 - Transient response of a Blumlein structure terminated with a plasma load.

To optimize the result, the parameters under consideration include the dielectric constant of the slow line $\varepsilon_{3}$, length of the transmission lines $L$, and characteristic impedance of the lines $Z_{0}$ which is related to the dimension, geometry, and material inside a transmission line. Figure 21 shows the plot of the output efficiency as a function of $\varepsilon_{3}$. The fixed parameters are $\varepsilon_{\mathrm{f}}=10.8, \mathrm{~L}=2.25 \mathrm{~m}$, and $Z_{0}=0.82 \Omega$. From the figure, we can see that the output efficiency increases as $\varepsilon_{\mathrm{z}}$ increases until the point where $\varepsilon_{\mathrm{z}}>9 \varepsilon_{\mathrm{f}}$. This result is what we expected it to be. The input energy is proportional to the dielectric constant of the material inside a transmission line. Therefore, as $\varepsilon_{\mathrm{s}}$ increases, the input energy also increases. However, the net voltage of the first output pulse will go to zero either at $t=L / v_{s}$ after a short circuit is applied if $\varepsilon_{\mathrm{s}}<9 \varepsilon_{\mathrm{f}}$, or at $t=3 \mathrm{~L} / \mathrm{v}_{\mathrm{f}}$ if $\varepsilon_{\mathrm{s}}>9 \varepsilon_{\mathrm{f}}$. That is, the output energy will stay constant after it reaches the point where $\varepsilon_{\mathrm{s}}=9 \varepsilon_{\mathrm{f}}$. Therefore, the efficiency will decrease as the result. From the figure, the maximum efficiency is $22.7 \%$.

For a Blumlein with a constant load, the output efficiency is not dependent on the length of the transmission lines. However, if the Blumlein is terminated with a dynamic load, the output efficiency is a function of the length of the transmission lines (Figure 22). The fixed parameters are $\varepsilon_{f}$ $=10.8, \varepsilon_{3}=95$, and $Z_{0}=0.82 \Omega$. As the length of the transmission line increases, the output efficiency also increases until it reaches $\mathrm{L}=2.3 \mathrm{~m}$, then the efficiency decreases afterwards. The maximum efficiency is $22.7 \%$ which occurs at a length $\mathrm{L}_{m}$, when $\mathrm{L}_{m} / \mathrm{v}$, is the time when the load impedance equals the characteristic impedance of the transmission line. This is also illustrated in Figure 23 which shows the plot of the output efficiency versus the characteristic impedance of the line. $\varepsilon_{\mathrm{f}}=10.8, \varepsilon_{\mathrm{s}}=95$, and $\mathrm{L}=2.25 \mathrm{~m}$ are the fixed parameters. The maximum output efficiency occurs when the characteristic impedance of the line is equal to the load impedance at the time, $L / v_{\mathbf{s}}$. 


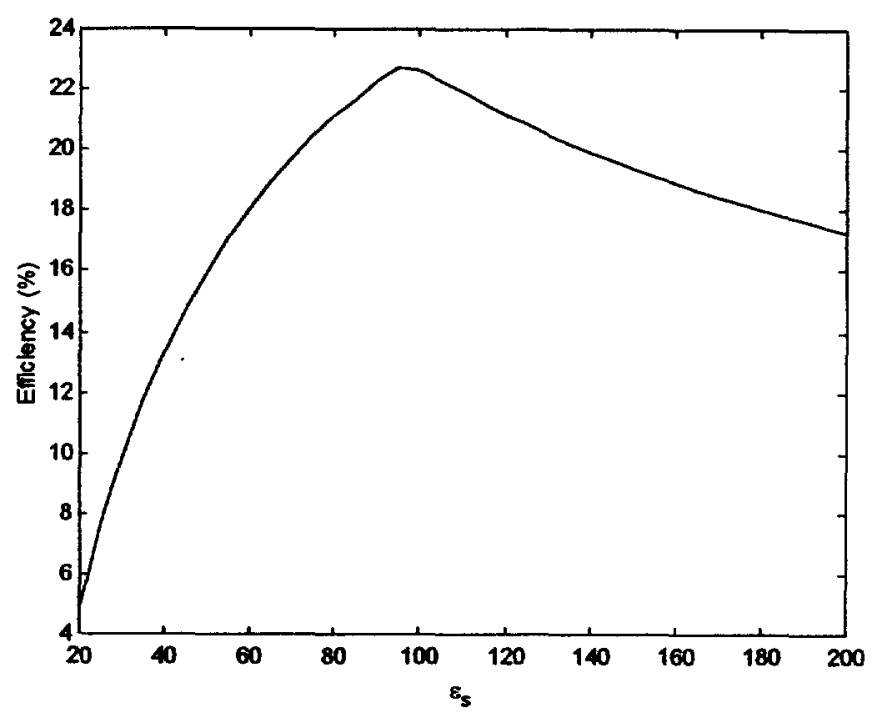

Figure 21 - Output efficiency versus the dielectric constant of the slow line $\varepsilon_{3}$. The dielectric constant of the fast line is 10.8. The characteristic impedance of the lines is $0.82 \Omega$. The length of the lines is $2.25 \mathrm{~m}$. The maximum efficiency for this case is $22.7 \%$ which occurs when $\varepsilon_{z}$ approaches $9 \varepsilon_{\text {f }}$.

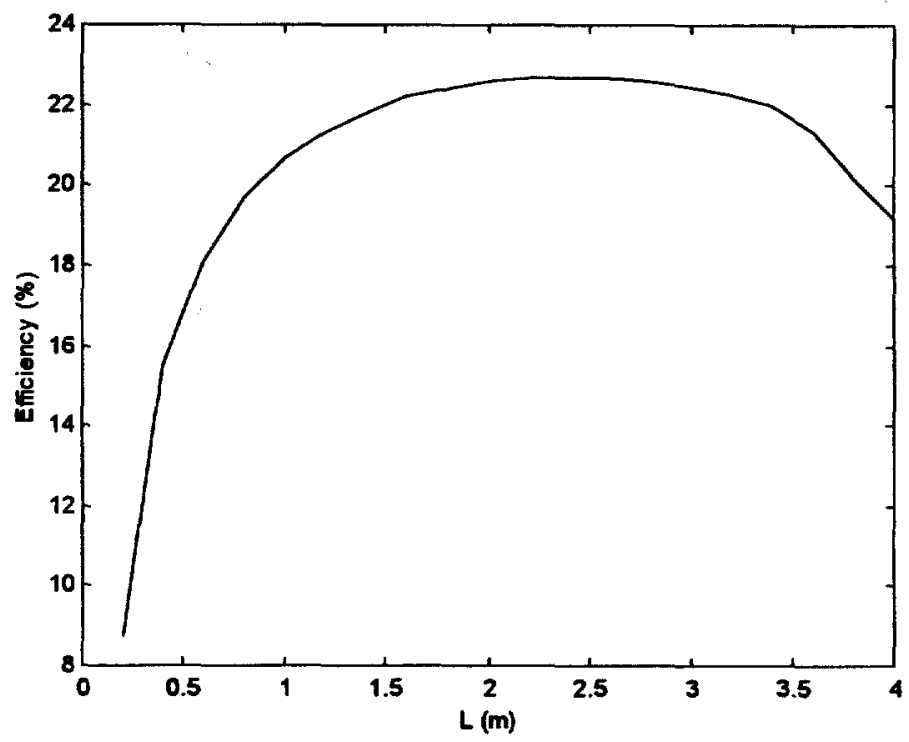

Figure 22 - Output efficiency versus the length of the transmission lines. The dielectric constants of the fast line and slow line are 10.8 and 95 , respectively. The characteristic impedance of the lines is $0.82 \mathrm{ohms}$. The maximum efficiency for this case is $22.7 \%$ which occurs when $\mathrm{L}$ is about $2.3 \mathrm{~m}$. 


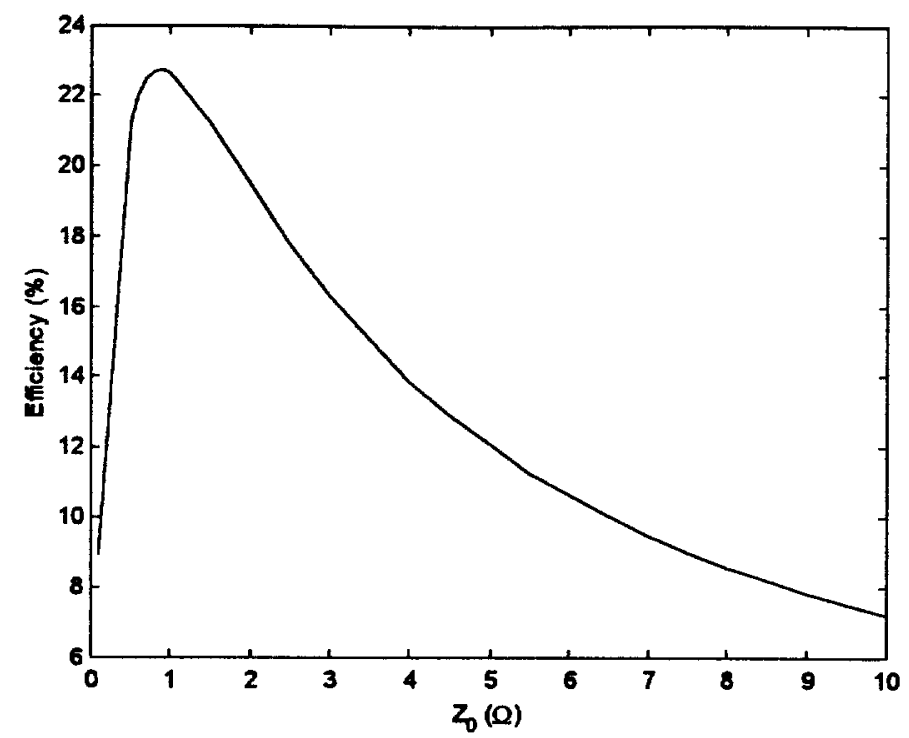

Figure 23 - Output efficiency versus the characteristic impedance of the line. The dielectric constants of the fast line and slow line are 10.8 and 95 , respectively. The length of the lines is $2.25 \mathrm{~m}$. The maximum efficiency for this case is $22.7 \%$ which occurs near $Z_{0}=0.8 \Omega$.

\section{Electric and magnetic coupling coefficients in the pulse-forming line array}

The pulse-forming transmission lines, which comprise the dielectric wall accelerator, can exhibit mutual coupling effects, which can modify the operation of the transmission line array. For this effort, the TEM electric and magnetic coupling coefficients are determined for a 2-D array of strip transmission lines using a 2-D finite element modeling code. The coupling coefficients can be used to determine an equivalent capacitance matrix or inductance matrix to be used in a circuit model for the coupled system. The array studied here consists of a stack of 20 transmission lines with alternating transmission line layers with each alternating layer having a different dielectric constant. The "fast" transmission line has a dielectric constant $\varepsilon_{f}$ and the "slow" transmission line has a dielectric constant $\varepsilon_{s}$. The configuration for a section of this geometry is shown in Figure 24 .

The width of the strips are $w=3 \mathrm{~cm}$. The fast line uses teflon as a dielectric constant with $\varepsilon_{\mathrm{f}}=$ $2.1, \mathrm{~d}_{\mathrm{f}}=1 \mathrm{~mm}$, and the slow line used duroid as the dielectric with $\varepsilon_{z}=10.8, \mathrm{~d}_{z}=2.27 \mathrm{~mm}$. The TEM impedance of a parallel plane transmission line follows from:

$$
Z_{f, s}=\sqrt{\frac{\mu_{0}}{\varepsilon_{f, s}}} \frac{d_{f, s}}{w}
$$




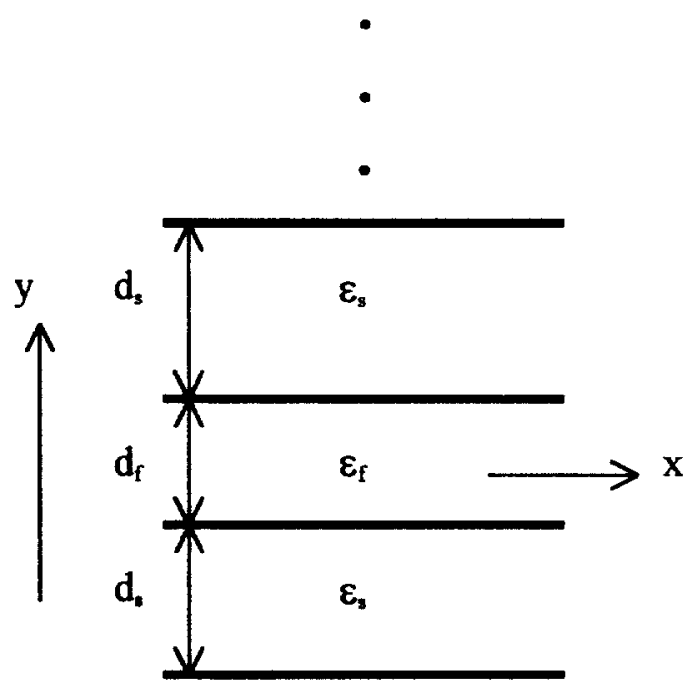

Figure 24 - Section of transmission line array showing the fast and slow lines. There are a total of 20 transmission lines in the stack, 10 slow and 10 fast.

For the geometry shown here the impedance of the transmission lines are both $8.7 \Omega$. For modeling the array, either a voltage is applied across, or a current is injected into the conductors defining the fast transmission line in the center of the stack (transmission line number 10) to determine the induced fields in the other transmission lines. These voltages and currents set up an electric field $E_{y}$ and a magnetic field $H_{x}$ in the fast line. The 2-D finite element solution determines the field solution within the entire transmission line array. The electric or magnetic coupling coefficient is defined as the ratio of the electric or magnetic field induced in the $j^{\text {th }}$ line to the drive electric or magnetic field. The induced charge on the transmission line conductors can be found by applying Gauss' law for the $f^{\text {th }}$ line

$$
Q_{j}=\oiint_{S_{j}} \mathbf{D} \cdot \mathbf{d} \mathbf{S}_{j}
$$

where $S_{j}$ represent the closed surface around the $f^{\text {th }}$ conductor. The circuit capacitances can be determined from

$$
Q=[C]
$$

where [C] represents the capacitance matrix and $Q$ and $V$ is the charge and voltage on the $f^{\text {th }}$ line. Similarly, the mutual inductance terms can be found from: 


$$
L_{i j}=\frac{\iint_{S} \mathbf{B}_{j} \cdot \mathbf{d} \mathbf{S}_{i}}{I_{j}}
$$

Figures 25 and 26 show the electric and magnetic coupling between the transmission lines and the driven line.

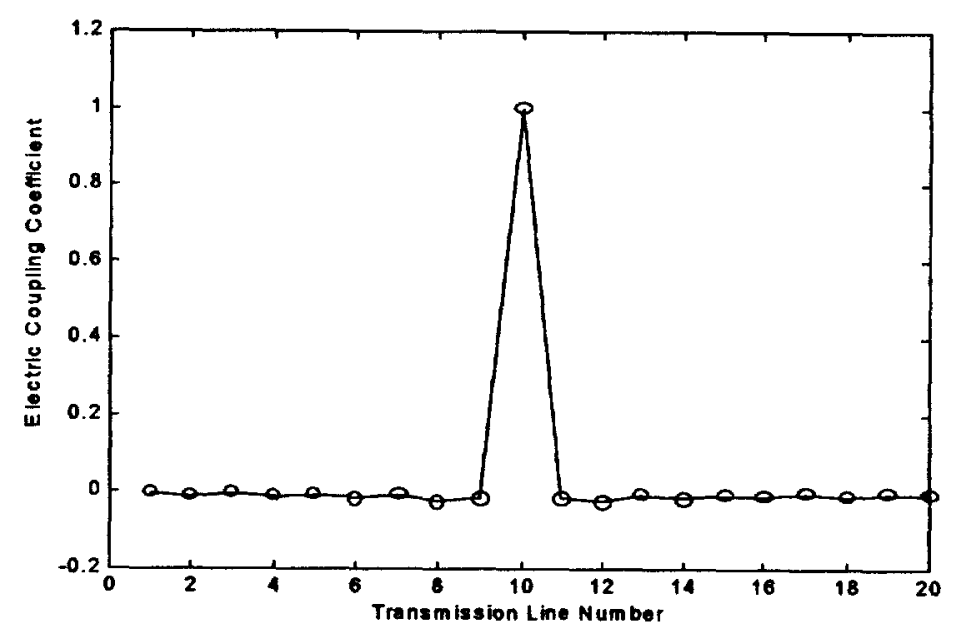

Figure 25 - Electric coupling for 20 layer stack

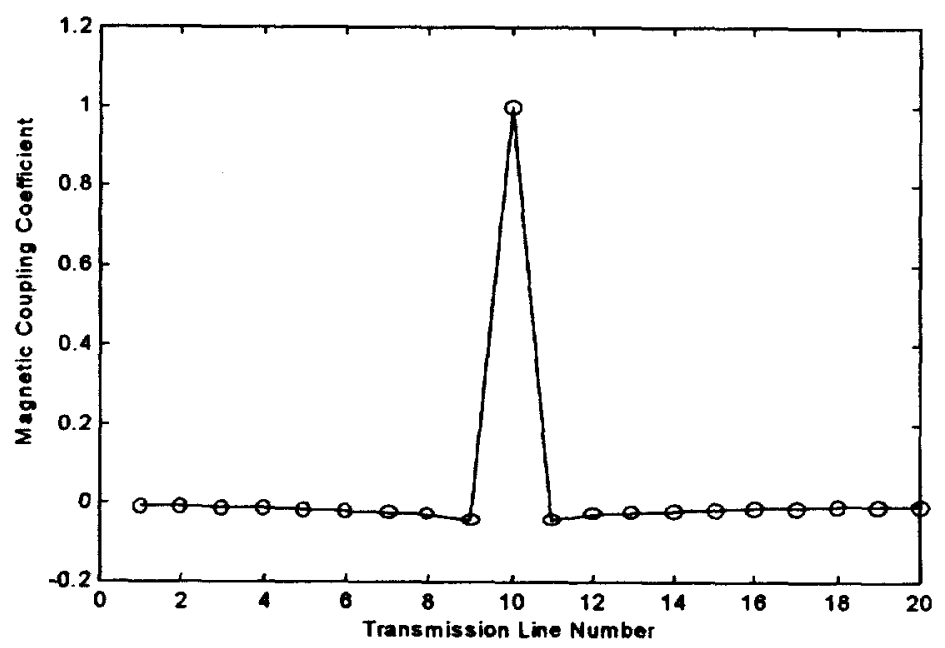

Figure 26 - Magnetic coupling for 20 layer stack 
Since the gaps between transmission lines is small compared to the width of the lines the mutual coupling due to the fringing fields in this case is very small $(<4 \%$ to the nearest neighbor transmission line).

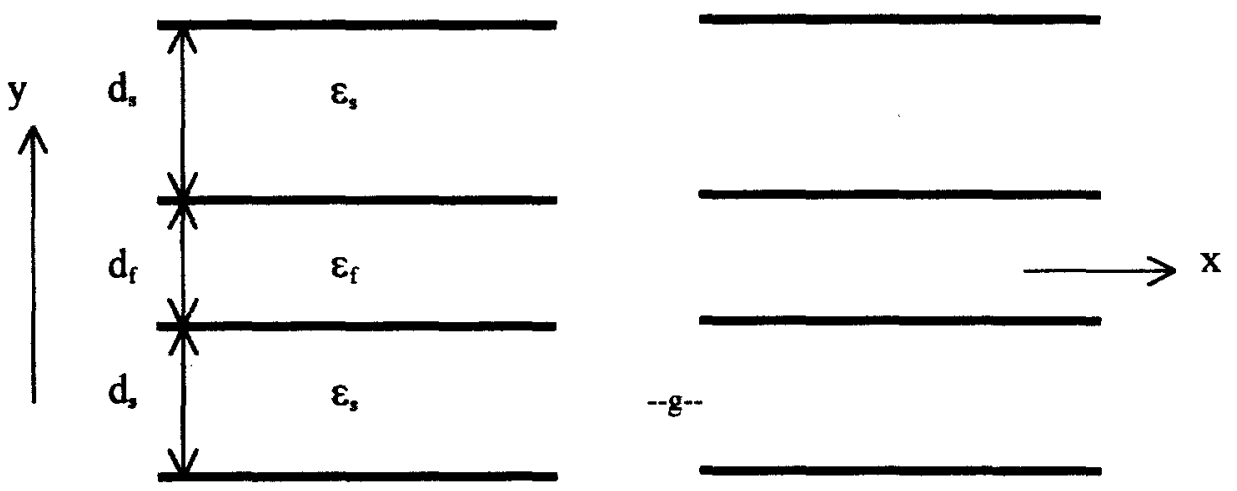

Stack 1

Stack 2

Figure 27. Transmission line array with 2 stacks of 20 layers.

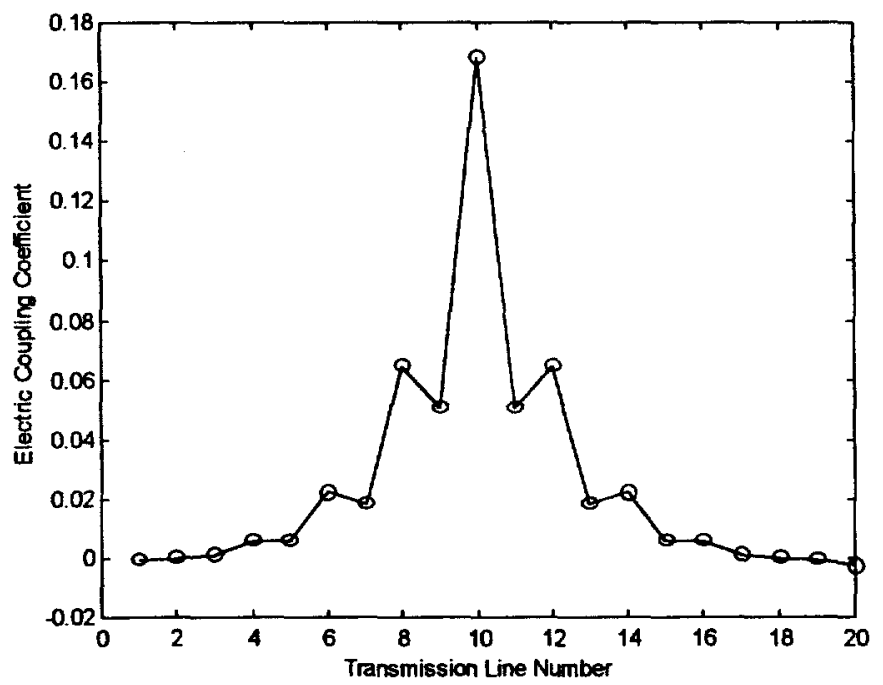

Figure 28 - Electric coupling from stack 1 to stack 2 for $\mathrm{g}=0.5 \mathrm{~mm}$ 


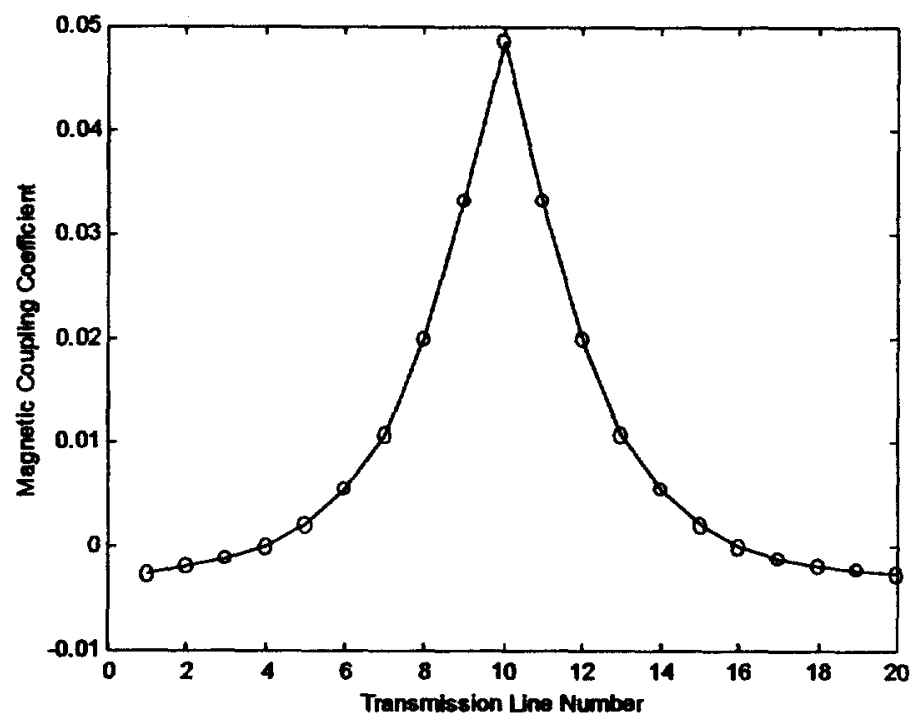

Figure 29 - Magnetic coupling from stack 1 to stack 2 for $g=0.5 \mathrm{~mm}$

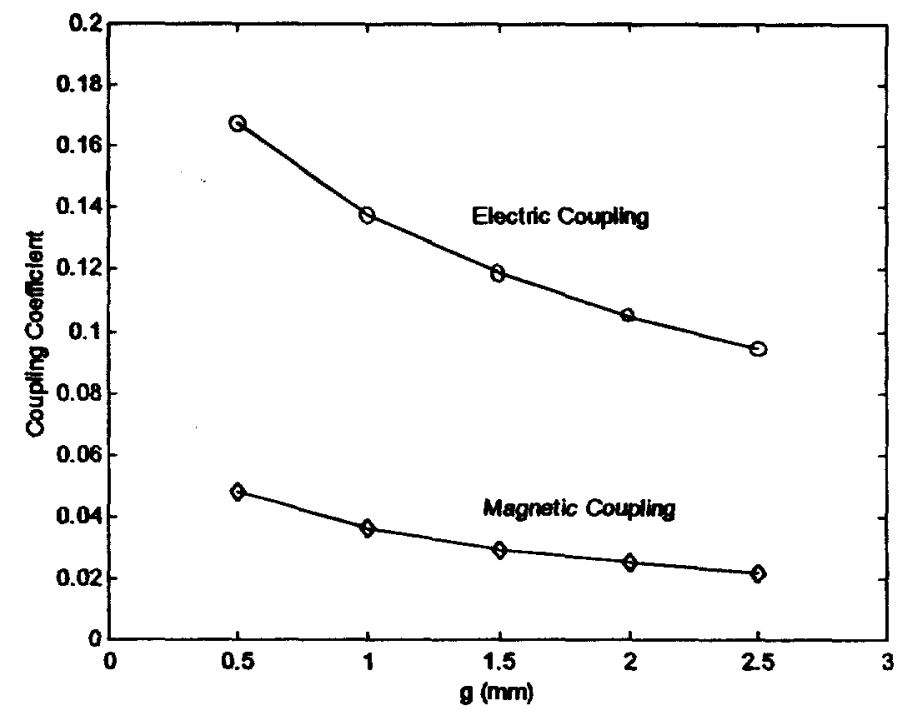

Figure 30 - Coupling between center layer on stack 1 to center layer on stack 2

In the dielectric wall accelerator several conductors may be located on each dielectric layer leading to a configuration such as that shown in Figure 27. In this geometry if the gap between adjacent layers is sufficiently close the mutual coupling between transmission lines can be substantially increased. In this case we still drive the center transmission line (line number 10) on stack number 1 and determine the coupling coefficients to the other transmission lines. The pa- 
rameters associated with the individual stacks are the same as before. We allow the gap between stacks to vary from $0.5 \mathrm{~mm}$ to $2.5 \mathrm{~mm}$.

Figures 28 and 29 show the electric and magnetic coupling into stack 2 with the center transmission line driven in stack 1 .

As can be seem in Figures 28-30 adding adjacent stacks in close proximity to each other can significantly increase the mutual coupling between transmission lines. Equivalent circuit parameters can be found from the field coupling coefficients to include in a coupled transmission line analysis of the structure.

\section{SUMMARY}

We are pursuing a new accelerator technology, the dielectric wall accelerator (DWA). This technology is potentially an ultra compact accelerator/pulsed power driver. This new accelerator relies on three new components: an ultra-high gradient insulator, an asymmetric Blumlein, and low jitter switches. In this report, we focused our attention on the first two components of the DWA system: the insulators and the asymmetric Blumlein.

First, we sought to develop the necessary design tools to model and scale the behavior of the high gradient insulator. To initiate this task, we concentrated on modeling the discharge processes (i.e., initiation and creation of the surface discharge). From our study of present models, we conclude that an accurate theoretical model of surface breakdown must not only include aspects of standard models, but must also treat photo-ionization during the long time scale evolution of the streamer discharge though the evaporated gas. That is, the tip of a propagating streamer is known to produce intense high energy radiation emission which we believe can lead to photoconduction in the insulator. As conduction in the insulator will strongly modify the voltage which drives the streamer discharge, accurate coupling of the streamer to the insulator were included. We showed initial calculations that were performed.

The electromagnetic characteristics of the HGI are due to perturbing the geometry of the resonant cavity. Careful incorporation of HGI's into an induction cell design should always lead to a much lower impedance than that of a conventional solid insulator. Further, as the higher voltage hold off of the HGI allow for narrower gaps and the transverse impedance scales directly with gap width, an additional factor of two reduction is expected.

Second, we performed circuit modeling to understand energy coupling to dynamic loads by the Asymmetric Blumlein. Our calculations show that reasonable coupling efficiencies can be realized. Further, we experimentally observe that these Blumlein structures, when stacked into a complete module, that the output voltage does not sum linearly (i.e., a lower than expected output voltage results). Thus we performed calculations to determine coupling coefficients of various geometries to understand this effect. 


\section{REFERENCES}

[1] G. J. Caporaso, "Induction Linacs and Pulsed Power," in Proceedings 1994 Joint Topical Course, Maui, HI (1994).

[2] J. Elizondo and A. Rodriguez, "Novel High Voltage Surface Flashover Insulator Technology," in Proceedings of the 1992, 15th International Symposium on Discharges and Electrical Insulation in Vacuum, Berlin, Germany (1992).

[3] S. Sampayan, et. al., "High Gradient Insulator Technology for the Dielectric Wall Accelerator," in Proceedings of the 1995 Particle Accelerator Conference, Dallas, TX (1995).

[4] S. Sampayan, et. al., "High Performance Insulator Structures for Accelerator Applications," Lawrence Livermore National Laboratory Report, UCRL-53868-96 (1996).

[5] S. C. Zhang, Department of Ceramic Engineering, University of Missouri, Rolla, MO, private communication.

[6] S. Sampayan, et. al., "Optically Induced Surface Flashover Switching for the Dielectric Wall Accelerator," in Proceedings of the 1995 Particle Accelerator Conference, Dallas, TX (1995).

[7] P. A. Vitello, et. al., "INDUCT '94: A Two-Dimensional Fluid Model of High Density Inductively Coupled Plasma Sources," Lawrence Livermore National Laboratory Report, UCRL-MA120465 (1995).

[8] A. Watson, "Pulsed Flashover in Vacuum," J. Appl. Phys, 38, 2019 (1967).

[9] G. Blaise, "Space-charge physics and the breakdown process," J. Appl. Phys., 77, 2916 (1995).

[10] J.M. Elizondo, M.L. Krogh, D. Smith, D. Stoltz, S.N. Wright, S.E. Sampayan, G.J. Caporaso, P. Vitello, N. Tishchenko, "Vacuum Surface Flashover and High Pressure Gas Streamers," (1997).

[11] T.L. Houck, et al., "Measured and Theoretical Characterization of the RF Properties of Stacked, High-Gradient Insulator Material" Proceedings of the 1997 IEEE Particle Accelerator Conference, Vancouver, BC, Canada, 12-16 May 1997.

[12] S. Eylon, T.L. Houck, et al., 'Longitudinal Impedance Measurements of an RK-TBA Induction Accelerating Gap" Proceedings of the 1997 IEEE Particle Accelerator Conference, Vancouver, BC, Canada, 12-16 May 1997. 


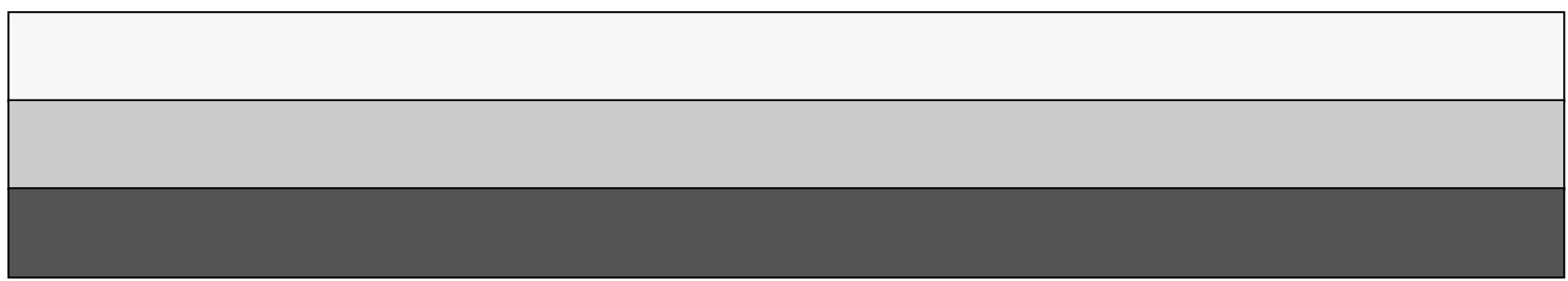

